\title{
Synthesis of Hydrophosphorylated Fullerene under Neutral Conditions
}

Hiroyuki Isobe, Ai-Jan Chen, Niclas Solin and Eiichi Nakamura

Department of Chemistry and ERATO (JST), The University of Tokyo, Hongo, Bunkyo-ku, Tokyo 113-0033, Japan

\section{Contents}

Experimental details and spectral data. .S1

NMR and mass spectra. .S8

\section{Experimental details and spectral data}

Spectral Measurement. IR spectra were obtained on an ASI Applied Systems REACT IR1000 equipped with an attenuated total reflection (ATR) instrument. NMR spectra were obtained on JEOL ECX-400 and ECA-500 spectrometers $\left({ }^{1} \mathrm{H}: 400\right.$ and $500 \mathrm{MHz} ;{ }^{13} \mathrm{C}: 100$ and $125 \mathrm{MHz}$ ). ${ }^{1} \mathrm{H}$ NMR spectra in $\mathrm{CDCl}_{3}$ were referenced internally to tetramethylsilane as a standard. ${ }^{13} \mathrm{C}$ NMR spectra in $\mathrm{CDCl}_{3}$ were referenced to the solvent resonance, and methyl, methylene, and methyne signals were assigned by DEPT spectra. Mass spectra were obtained on JEOL JMS-T100LC (AccuTOF; APCI/ESI-TOF MS).

Synthesis of diphenyl[9-hydro $\left(C_{60}-I_{h}\right)[5,6]$ fulleren-1(9H)-yl]phosphine oxide by the reaction of [60]fullerene and diphenylphosphine oxide (Table 1, entry 1-3): To a solution of $\mathrm{C}_{60}(1.00 \mathrm{~g}, 1.39 \mathrm{mmol})$ in chlorobenzene $(400 \mathrm{~mL})$ was added DMSO $(100 \mathrm{~mL})$. To the solution was then added diphenylphosphine oxide (282 mg, $1.39 \mathrm{mmol} ; 1.0$ equiv), and the mixture was stirred under air at ambient temperature. HPLC analysis (Bucky-prep column, eluent: $i$-PrOH/toluene $=3 / 7$ ) of the mixture showed that yield of mono-adduct reached its maximum after 48 hours. The mixture was washed with brine, and the organic layer was dried over anhydrous $\mathrm{Na}_{2} \mathrm{SO}_{4}$. The crude solution was purified by silica gel column chromatography (100 g, eluent: chlorobenzene and 10\% ethyl acetate/toluene) to give $\mathrm{C}_{60}(280 \mathrm{mg}, 28 \%$ recovery) and the title compound $(738 \mathrm{mg}, 54 \%)$ as an analytically pure brown powder. The product was identical with an authentic sample by HPLC, IR, NMR and MS analyses. ${ }^{1}$ The reaction was also carried out in a mixed solvent containing

1. Yamago, S.; Yanagawa, M.; Mukai, H.; Nakamura E. Tetrahedron 1996, 52, 5091-5092; Yamago, S.; Yanagawa, M.; Nakamura E. J. Chem. Soc., Chem. Commun. 1994, 2093-2094. 
$20 \% \mathrm{v} / \mathrm{v}$ of HMPA or DMF to obtain the title compound in 60\% yield (reaction time: $6 \mathrm{~h}$, $\mathrm{C}_{60}$ recovery: $30 \%$ ) and $47 \%$ yield (reaction time: $96 \mathrm{~h}, \mathrm{C}_{60}$ recovery $34 \%$ ), respectively.

Synthesis of diphenyl[9-hydro $\left(\mathrm{C}_{60}-I_{h}\right)[5,6]$ fulleren-1(9H)-yl]phosphine oxide by the reaction of [60]fullerene and diphenylphosphine (Table 1, entry 4): To a solution of $\mathrm{C}_{60}$ (50 mg, $69.4 \mu \mathrm{mol})$ in chlorobenzene $(20 \mathrm{~mL})$ was added DMSO $(5 \mathrm{~mL})$. To the solution was then added diphenlyphosphine $(72.5 \mu \mathrm{L}, 417 \mu \mathrm{mol} ; 6.0$ equiv), and the mixture was stirred under air at ambient temperature. HPLC analysis (ODS column, eluent: $i$ - $\mathrm{PrOH} /$ toluene $=7 / 3$ ) of the mixture showed that $\mathrm{C}_{60}$ was completely consumed after 12 hours. The mixture was diluted with toluene up to twice amount and washed with brine. The organic layer was dried over anhydrous $\mathrm{MgSO}_{4}$, and concentrated in vacuo. The crude material was purified by silica gel column chromatography ( $5 \mathrm{~g}$, eluent: toluene and $20 \%$ ethyl acetate/toluene) to give the title compound $(39.7 \mathrm{mg}, 57 \%)$ as an analytically pure brown powder. The product was identical with an authentic sample by HPLC, IR, NMR and MS analyses. The reaction under atmospheric pressure of nitrogen gave essentially an identical result.

\section{Synthesis of di(4-methoxylphenyl)[9-hydro $\left(C_{60}-I_{h}\right)[5,6]$ fulleren-1(9H)-yl]phosphine} oxide by the reaction of [60]fullerene and di(4-methoxylphenyl)phosphine oxide (Table 1, entry 5): To a solution of $\mathrm{C}_{60}(51.4 \mathrm{mg}, 71.4 \mu \mathrm{mol})$ in chlorobenzene $(20 \mathrm{~mL})$ was added DMSO $(5 \mathrm{~mL})$. To the solution was then added di(4-methoxylphenyl)phosphine oxide (18.6 mg, $71.4 \mu \mathrm{mol} ; 1.0$ equiv), and the mixture was stirred under air at ambient temperature. HPLC analysis (Bucky-prep column, eluent: $i$-PrOH/toluene $=3 / 7$ ) of the mixture showed that yield of mono-adduct reached its maximum after 48 hours. The mixture was washed with brine, and the organic layer was dried over anhydrous $\mathrm{Na}_{2} \mathrm{SO}_{4}$. The crude solution was purified by silica gel column chromatography (5 $\mathrm{g}$, eluent: chlorobenzene and $10 \%$ ethyl acetate/toluene) to give $\mathrm{C}_{60}(19.5 \mathrm{mg}, 38 \%$ recovery) and the title compound (31.6 mg, 50\%) as an analytically pure brown powder. IR (powder) 2949, 2903, 2837, 1590, 1463, 1424, 1390, 1289, 1181, 1112, 1023, 822.2, 775.9, $706.4 \mathrm{~cm}^{-1}$; ${ }^{31} \mathrm{P}$ NMR

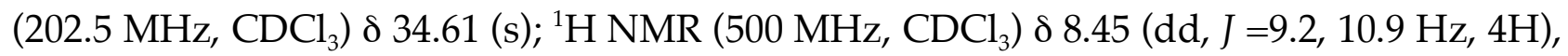
$7.18(\mathrm{dd}, J=2.9,9.2 \mathrm{~Hz}, 4 \mathrm{H}), 7.12(\mathrm{~d}, J=25.8 \mathrm{~Hz}, 1 \mathrm{H}), 3.92(\mathrm{~s}, 6 \mathrm{H}) ;{ }^{13} \mathrm{C} \mathrm{NMR}(125 \mathrm{MHz}$, $\left.\mathrm{CDCl}_{3}\right) \delta 55.48\left(\mathrm{CH}_{3}\right), 57.20\left(\mathrm{~d}, J_{\mathrm{C}-\mathrm{P}}=3.0 \mathrm{~Hz}\right), 71.27\left(\mathrm{~d}, J_{\mathrm{C}-\mathrm{P}}=66.8 \mathrm{~Hz}\right), 114.54\left(\mathrm{~d}, J_{\mathrm{C}-\mathrm{P}}=13.1\right.$ $\mathrm{Hz}, \mathrm{CH}), 120.13\left(\mathrm{~d}, J_{\mathrm{C}-\mathrm{P}}=105.5 \mathrm{~Hz}, \mathrm{CH}\right), 135.09$ (d, J $\left.J_{\text {C-P }}=9.6 \mathrm{~Hz}, \mathrm{CH}\right), 135.71$ (d, J $J_{\text {C-P }}=1.8$ $\mathrm{Hz}), 137.08\left(\mathrm{~d}, J_{\mathrm{C}-\mathrm{P}}=5.4 \mathrm{~Hz}\right), 139.79,140.43,141.51,141.60,141.62,141.76,141.88,142.13$, 142.16 , 142.58, 142.71, 143.31, 144.37, 144.77, 145.47, 145.49, 145.62, 145.86, 146.30, 146.38, $146.45,146.52,147.02,147.22,147.27,147.56\left(\mathrm{~d}, J_{\mathrm{C}-\mathrm{P}}=1.8 \mathrm{~Hz}\right), 149.98\left(\mathrm{~d}, J_{\mathrm{C}-\mathrm{P}}=6.6 \mathrm{~Hz}\right), 152.73$ 
$\left(\mathrm{d}, J_{\mathrm{C}-\mathrm{P}}=4.8 \mathrm{~Hz}\right), 163.25\left(\mathrm{~d}, J_{\mathrm{C}-\mathrm{P}}=3.0 \mathrm{~Hz}, \mathrm{CH}\right.$ ); HRMS (APCI-TOF, negative) $\mathrm{m} / \mathrm{z}$ calcd for $\mathrm{C}_{74} \mathrm{H}_{15} \mathrm{O}_{3} \mathrm{P}[\mathrm{M}-\mathrm{H}]^{-}$981.0681, found: 981.0681 .

Synthesis of di(3,5-di-t-butylphenyl)[9-hydro $\left(\mathrm{C}_{60}-I_{\mathrm{h}}\right)[5,6]$ fulleren-1(9H)-yl]phosphine oxide by the reaction of [60]fullerene and di(3,5-di-t-butylphenyl)phosphine oxide (Table 1, entry 6): To a solution of $\mathrm{C}_{60}(51.2 \mathrm{mg}, 71.1 \mu \mathrm{mol})$ in chlorobenzene $(20 \mathrm{~mL})$ was added DMSO (5 mL). To the solution was then added di(3,5-di-t-butylphenyl)phosphine oxide (30.3 mg, $71.1 \mu \mathrm{mol} ; 1.0$ equiv), and the mixture was stirred under air at ambient temperature. HPLC analysis (Bucky-prep column, eluent: $i-\mathrm{PrOH} /$ toluene $=3 / 7$ ) of the mixture showed that yield of mono-adduct reached its maximum after 18 hours. The mixture was washed with brine, and the organic layer was dried over anhydrous $\mathrm{Na}_{2} \mathrm{SO}_{4}$. The crude solution was purified by silica gel column chromatography (5 g, eluent: chlorobenzene and $10 \%$ ethyl acetate/toluene) to give $\mathrm{C}_{60}(19.3 \mathrm{mg}, 38 \%$ recovery) and the title compound ( $44.6 \mathrm{mg}, 55 \%$ ) as an analytically pure brown powder. IR (powder) 2949, 2903, 2860, 1590, 1463, 1424, 1393, 1363, 1247, 1200, 1146, 895.5, 868.5, 795.2, 706.4 $\mathrm{cm}^{-1} ;{ }^{31} \mathrm{P}$ NMR (202.5 MHz, $\left.\mathrm{CDCl}_{3}\right) \delta 36.91$ (s); ${ }^{1} \mathrm{H}$ NMR $\left(500 \mathrm{MHz}, \mathrm{CDCl}_{3}\right) \delta 8.37(\mathrm{dd}, J=1.7,12.0$ $\mathrm{Hz}, 4 \mathrm{H}), 7.72(\mathrm{~s}, 2 \mathrm{H}), 7.11(\mathrm{~d}, J=25.2 \mathrm{~Hz}, 1 \mathrm{H}), 1.38(\mathrm{~s}, 36 \mathrm{H}) ;{ }^{13} \mathrm{C} \mathrm{NMR}\left(125 \mathrm{MHz}, \mathrm{CDCl}_{3}\right) \delta$ $31.38\left(\mathrm{CH}_{3}\right), 35.28\left(\mathrm{CH}_{3}\right), 56.95\left(\mathrm{~d}, J_{\mathrm{C}-\mathrm{P}}=3.0 \mathrm{~Hz}\right), 71.12\left(\mathrm{~d}, J_{\mathrm{C}-\mathrm{P}}=64.4 \mathrm{~Hz}\right), 127.09\left(\mathrm{~d}, J_{\mathrm{C}-\mathrm{P}}=2.4\right.$ $\mathrm{Hz}, \mathrm{CH}), 127.59\left(\mathrm{~d}, J_{\mathrm{C}-\mathrm{P}}=9.0 \mathrm{~Hz}, \mathrm{CH}\right), 128.06\left(\mathrm{~d}, J_{\mathrm{C}-\mathrm{P}}=97.2 \mathrm{~Hz}, \mathrm{CH}\right), 135.84\left(\mathrm{~d}, J_{\mathrm{C}-\mathrm{P}}=1.8 \mathrm{~Hz}\right)$, $136.81\left(\mathrm{~d}, J_{\mathrm{C}-\mathrm{P}}=4.8 \mathrm{~Hz}\right), 139.28\left(\mathrm{~d}, J_{\mathrm{C}-\mathrm{P}}=1.2 \mathrm{~Hz}\right), 140.38,141.36,141.48,141.50,141.75,141.87$, $142.02,142.09,142.57,142.69,143.30,144.40,144.72,145.31,145.33,145.45,145.52,145.58$, 145.95, 146.29, 146.35, 146.45, 146.53, 147.13, 147.21, 147.25, 147.47 (d, J J $\left.\mathrm{C}_{\mathrm{P}}=1.2 \mathrm{~Hz}\right), 150.47$ $\left(\mathrm{d}, J_{\mathrm{C}-\mathrm{P}}=6.6 \mathrm{~Hz}\right), 151.35\left(\mathrm{~d}, J_{\mathrm{C}-\mathrm{P}}=11.3 \mathrm{~Hz}, \mathrm{CH}\right), 153.10\left(\mathrm{~d}, J_{\mathrm{C}-\mathrm{P}}=4.8 \mathrm{~Hz}\right.$ ); HRMS (APCI-TOF, negative) $\mathrm{m} / \mathrm{z}$ calcd for $\mathrm{C}_{88} \mathrm{H}_{43} \mathrm{OP}[\mathrm{M}-\mathrm{H}]^{-} 1145.2973$, found: 1145.2979 .

Synthesis of di(4-fluorophenyl)[9-hydro $\left(\mathrm{C}_{60}-\mathrm{I}_{\mathrm{h}}\right)[5,6]$ fulleren-1(9H)-yl]phosphine oxide by the reaction of [60]fullerene and di(4-fluorophenyl)phosphine oxide (Table 1, entry 7): To a solution of $\mathrm{C}_{60}(51.3 \mathrm{mg}, 69.8 \mu \mathrm{mol})$ in chlorobenzene $(20 \mathrm{~mL})$ was added DMSO (5 $\mathrm{mL}$ ). To the solution was then added di(4-methoxylphenyl)phosphine oxide (16.6 mg, 69.8 $\mu$ mol; 1.0 equiv), and the mixture was stirred under air at ambient temperature. HPLC analysis (Bucky-prep column, eluent: $i-\mathrm{PrOH} /$ toluene $=3 / 7$ ) of the mixture showed that yield of mono-adduct reached its maximum after 60 hours. The mixture was washed with brine, and the organic layer was dried over anhydrous $\mathrm{Na}_{2} \mathrm{SO}_{4}$. The crude solution was purified by silica gel column chromatography $(5 \mathrm{~g}$, eluent: chlorobenzene and $10 \%$ ethyl acetate/toluene) to give $\mathrm{C}_{60}(28.1 \mathrm{mg}, 56 \%$ recovery) and the title compound (24.8 $\mathrm{mg}$, $37 \%$ ) as an analytically pure brown powder. IR (powder) $2981,2879,1588,1495,1426,1395$, 
1233, 1198, 1158, 1111, 1094, 1013, 897.2, 827.8, $750.6 \mathrm{~cm}^{-1} ;{ }^{31} \mathrm{P}$ NMR $\left(202.5 \mathrm{MHz}, \mathrm{CDCl}_{3}\right) \delta$ 32.78 (s); ${ }^{1} \mathrm{H}$ NMR (500 MHz, $\left.\mathrm{CDCl}_{3}\right) \delta 8.55$ (br, 4H), $7.40(\mathrm{br}, 4 \mathrm{H}), 7.09(\mathrm{~d}, J=26.7 \mathrm{~Hz}, 1 \mathrm{H})$; ${ }^{13} \mathrm{C}$ NMR $\left(125 \mathrm{MHz}, \mathrm{CDCl}_{3}\right) \delta 56.93\left(\mathrm{~d}, J_{\mathrm{C}-\mathrm{P}}=3.0 \mathrm{~Hz}\right), 70.53\left(\mathrm{~d}, J_{\mathrm{C}-\mathrm{P}}=69.2 \mathrm{~Hz}\right), 116.66(\mathrm{dd}$, $\left.J_{\mathrm{C}-\mathrm{P}}=12.5 \mathrm{~Hz}, J_{\mathrm{C}-\mathrm{F}}=20.9 \mathrm{~Hz}, \mathrm{CH}\right), 124.68\left(\mathrm{dd}, J_{\mathrm{C}-\mathrm{P}}=102.0 \mathrm{~Hz}, J_{\mathrm{C}-\mathrm{F}}=3.6 \mathrm{~Hz}, \mathrm{CH}\right), 135.47(\mathrm{~d}$, $\left.J_{\mathrm{C}-\mathrm{P}}=1.8 \mathrm{~Hz}\right), 135.77\left(\mathrm{dd}, J_{\mathrm{C}-\mathrm{P}}=9.0, J_{\mathrm{C}-\mathrm{F}}=9.0 \mathrm{~Hz}, \mathrm{CH}\right), 137.28\left(\mathrm{~d}, J_{\mathrm{C}-\mathrm{P}}=4.8 \mathrm{~Hz}\right), 139.82,140.57$, 141.49, 141.51, 141.54, 141.80, 141.86, 142.12, 142.16, 142.64, 142.80, 143.36, 144.30, 144.80, 145.50, 145.52, 145.61, 145.70, 145.78, 146.38, 146.43, 146.48, 146.60, 146.81, 147.07, 147.28, $148.82\left(\mathrm{~d}, J_{\mathrm{C}-\mathrm{P}}=7.2 \mathrm{~Hz}\right), 152.09\left(\mathrm{~d}, J_{\mathrm{C}-\mathrm{P}}=4.8 \mathrm{~Hz}\right) 165.85\left(\mathrm{dd} J_{\mathrm{C}-\mathrm{P}}=3.0 \mathrm{~Hz}, J_{\mathrm{C}-\mathrm{F}}=254.6 \mathrm{~Hz}\right.$, $\mathrm{CH}$ ); HRMS (APCI-TOF, negative) $\mathrm{m} / \mathrm{z}$ calcd for $\mathrm{C}_{72} \mathrm{H}_{9} \mathrm{~F}_{2} \mathrm{OP}[\mathrm{M}-\mathrm{H}]^{-}$957.0281, found: 957.0274.

\section{Synthesis of methyl(phenyl) [9-hydro $\left(\mathrm{C}_{60}-I_{\mathrm{h}}\right)[5,6]$ fulleren-1(9H)-yl]phosphine oxide by} the reaction of [60]fullerene and methyl(phenyl)phosphine oxide (Table 1, entry 8): To a solution of $\mathrm{C}_{60}(50.1 \mathrm{mg}, 69.6 \mu \mathrm{mol})$ in chlorobenzene $(20 \mathrm{~mL})$ was added DMSO $(5 \mathrm{~mL})$. To the solution was then added methyl(phenyl)phosphine oxide (10.0 mg, $69.6 \mu \mathrm{mol} ; 1.0$ equiv), and the mixture was stirred under air at ambient temperature. HPLC analysis (Bucky-prep column, eluent: $i-\mathrm{PrOH} /$ toluene $=3 / 7$ ) of the mixture showed that yield of mono-adduct reached its maximum after 31 hours. The mixture was washed with brine, and the organic layer was dried over anhydrous $\mathrm{Na}_{2} \mathrm{SO}_{4}$. The crude solution was purified by silica gel column chromatography ( $5 \mathrm{~g}$, eluent: chlorobenzene and $10 \%$ ethyl acetate/toluene) to give $\mathrm{C}_{60}(29.0 \mathrm{mg}, 58 \%$ recovery) and the title compound (18.5 mg, $31 \%$ ) as an analytically pure brown powder. IR (powder) 2960, 2917, 2861, 1600, 1451, 1302, 1285, 1191, 1177, 1111, 922.3, 903.0, 872.1, 860.6, 827.8, 740.9, $690.8 \mathrm{~cm}^{-1}$; ${ }^{31} \mathrm{P}$ NMR (202.5 $\left.\mathrm{MHz}, \mathrm{CDCl}_{3}\right) \delta 42.53(\mathrm{~s}) ;{ }^{1} \mathrm{H}$ NMR $\left(500 \mathrm{MHz}, \mathrm{CDCl}_{3}\right) \delta 8.30(\mathrm{dd}, J=7.4,11.5 \mathrm{~Hz}, 2 \mathrm{H}), 7.30$ $(\mathrm{m}, 3 \mathrm{H}), 7.24(\mathrm{~d}, J=30.9 \mathrm{~Hz}, 1 \mathrm{H}), 2.69(\mathrm{~d}, J=13.2 \mathrm{~Hz}, 3 \mathrm{H}) ;{ }^{13} \mathrm{C}$ NMR $\left(100 \mathrm{MHz}, \mathrm{CDCl}_{3}\right) \delta$ $12.77\left(\mathrm{~d}, J_{\mathrm{C}-\mathrm{P}}=71.8 \mathrm{~Hz}, \mathrm{CH}_{3}\right), 55.94\left(\mathrm{~d}, J_{\mathrm{C}-\mathrm{P}}=2.5 \mathrm{~Hz}\right), 69.87\left(\mathrm{~d}, J_{\mathrm{C}-\mathrm{P}}=65.8 \mathrm{~Hz}\right), 128.97\left(\mathrm{~d}, J_{\mathrm{C}-\mathrm{P}}\right.$ $=11.6 \mathrm{~Hz}, \mathrm{CH}), 129.02\left(\mathrm{~d}, J_{\mathrm{C}-\mathrm{P}}=130.0 \mathrm{~Hz}\right), 132.63\left(\mathrm{~d}, J_{\mathrm{C}-\mathrm{P}}=8.6 \mathrm{~Hz}, \mathrm{CH}\right), 133.35\left(\mathrm{~d}, J_{\mathrm{C}-\mathrm{P}}=2.9\right.$ $\mathrm{Hz}, \mathrm{CH}), 135.41\left(\mathrm{~d}, J_{\mathrm{C}-\mathrm{P}}=2.5 \mathrm{~Hz}\right), 135.62\left(\mathrm{~d}, J_{\mathrm{C}-\mathrm{P}}=1.7 \mathrm{~Hz}\right), 137.04\left(\mathrm{~d}, J_{\mathrm{C}-\mathrm{P}}=1.9 \mathrm{~Hz}\right), 137.09(\mathrm{~d}$, $\left.J_{\mathrm{C}-\mathrm{P}}=1.5 \mathrm{~Hz}\right), 140.12,140.17\left(\mathrm{~d}, J_{\mathrm{C}-\mathrm{P}}=1.2 \mathrm{~Hz}\right), 140.51,141.58,141.54,141.59,141.75,141.76$, 141.77, 141.79, 141.86, 141.91, 142.03, 142.11, 142.16, 142.63, 142.75, 143.31, 143.36, 144.31 (d, $\left.J_{\text {C-P }}=1.7 \mathrm{~Hz}\right), 144.39,144.77,144.80,145.43,145.47,145.50,145.56,145.58,145.59,145.63$, $145.70,145.79,146.36,146.40,146.42,146.49,146.54\left(\mathrm{~d}, J_{\mathrm{C}-\mathrm{P}}=1.0 \mathrm{~Hz}\right), 156.58\left(\mathrm{~d}, J_{\mathrm{C}-\mathrm{P}}=1.0 \mathrm{~Hz}\right)$, $147.21,147.29\left(\mathrm{~d}, J_{\mathrm{C}-\mathrm{P}}=2.5 \mathrm{~Hz}\right), 149.32\left(\mathrm{~d}, J_{\mathrm{C}-\mathrm{P}}=3.8 \mathrm{~Hz}\right), 152.12,152.31\left(\mathrm{~d}, J_{\mathrm{C}-\mathrm{P}}=4.6 \mathrm{~Hz}\right)$; HRMS (APCI-TOF, negative) $\mathrm{m} / \mathrm{z}$ calcd for $\mathrm{C}_{67} \mathrm{H}_{9} \mathrm{OP}[\mathrm{M}-\mathrm{H}]^{-}$859.0313, found: 859.0311. 
Synthesis of ethyl phenyl[9-hydro $\left(C_{60}-I_{h}\right)[5,6]$ fulleren-1(9H)-yl]phosphinate by the reaction of [60]fullerene and ethyl phenylphosphinate (Table 1, entry 9): To a solution of $\mathrm{C}_{60}(49.7 \mathrm{mg}, 69.0 \mu \mathrm{mol})$ in chlorobenzene $(20 \mathrm{~mL})$ was added DMSO $(5 \mathrm{~mL})$. To the solution was then added ethyl phenylphosphinate (141 mg, $828 \mu \mathrm{mol} ; 12.0$ equiv), and the mixture was stirred under air at $80{ }^{\circ} \mathrm{C}$. HPLC analysis (Bucky-prep column, eluent: $i$-PrOH/toluene $=3 / 7$ ) of the mixture showed that yield of mono-adduct reached its maximum after 24 hours. The mixture was washed with brine, and the organic layer was dried over anhydrous $\mathrm{Na}_{2} \mathrm{SO}_{4}$. The crude solution was purified by silica gel column chromatography (5 g, eluent: chlorobenzene and $10 \%$ ethyl acetate/toluene) to give $\mathrm{C}_{60}$ $(7.80 \mathrm{mg}, 16 \%$ recovery) and the title compound $(28.6 \mathrm{mg}, 46 \%)$ as an analytically pure brown powder. IR (powder) 2964, 2918, 2849, 1590, 1463, 1428, 1386, 1227, 1181, 1119, 1015, 949.6, 903.2, 799.0, 752.7, 725.7, $690.9 \mathrm{~cm}^{-1} ;{ }^{31} \mathrm{P} \mathrm{NMR}\left(202.5 \mathrm{MHz}, \mathrm{CDCl}_{3}\right) \delta 38.79$ (s); ${ }^{1} \mathrm{H}$ $\operatorname{NMR}\left(500 \mathrm{MHz}, \mathrm{CDCl}_{3}\right) \delta 8.30(\mathrm{ddd}, J=1.2,7.5,10.9 \mathrm{~Hz}, 2 \mathrm{H}), 7.73(\mathrm{dd}, J=1.2,7.5 \mathrm{~Hz}, 1 \mathrm{H})$, $7.66(\mathrm{dt}, J=4.0,7.5 \mathrm{~Hz}, 2 \mathrm{H}), 7.29(\mathrm{~d}, J=27.5 \mathrm{~Hz}, 1 \mathrm{H}), 4.79(\mathrm{~m}, 1 \mathrm{H}), 4.60(\mathrm{~m}, 1 \mathrm{H}), 1.67(\mathrm{t}, J=$ $6.9 \mathrm{~Hz}, 3 \mathrm{H}) ;{ }^{13} \mathrm{C}$ NMR $\left(125 \mathrm{MHz} \mathrm{CDCl}_{3}\right) \delta 16.92\left(\mathrm{~d}, J_{\mathrm{C}-\mathrm{P}}=6.0 \mathrm{~Hz}, \mathrm{CH}_{3}\right), 55.99\left(\mathrm{~d}, J_{\mathrm{C}-\mathrm{P}}=1.8\right.$ $\mathrm{Hz}), 63.78\left(\mathrm{~d}, J_{\mathrm{C}-\mathrm{P}}=6.0 \mathrm{~Hz}, \mathrm{CH}_{2}\right), 68.93\left(\mathrm{~d}, J_{\mathrm{C}-\mathrm{P}}=100.2 \mathrm{~Hz}\right), 126.7\left(\mathrm{~d}, J_{\mathrm{C}-\mathrm{P}}=130 \mathrm{~Hz}\right), 128.86(\mathrm{~d}$, $\left.J_{\text {C-P }}=12.5 \mathrm{~Hz}, \mathrm{CH}\right), 133.76\left(\mathrm{~d}, J_{\mathrm{C}-\mathrm{P}}=3.0 \mathrm{~Hz}, \mathrm{CH}\right), 134.13\left(\mathrm{~d}, J_{\mathrm{C}-\mathrm{P}}=8.9 \mathrm{~Hz}, \mathrm{CH}\right), 135.51\left(\mathrm{~d}, J_{\mathrm{C}-\mathrm{P}}\right.$ $=2.4 \mathrm{~Hz}), 135.75\left(\mathrm{~d}, J_{\mathrm{C}-\mathrm{P}}=2.4 \mathrm{~Hz}\right), 135.60\left(\mathrm{~d}, J_{\mathrm{C}-\mathrm{P}}=4.8 \mathrm{~Hz}\right), 135.64\left(\mathrm{~d}, J_{\mathrm{C}-\mathrm{P}}=5.4 \mathrm{~Hz}\right), 139.94$, $140.04,140.47,141.48,141.60,141.73,141.94,142.07,142.12,142.59,142.68,143.26\left(\mathrm{~d}, J_{\text {C-P }}\right.$ $=3.6 \mathrm{~Hz}), 144.36,144.43,144.72,145.44,145.49$, 145.58, 145.87, 145.90, 146.30, 146.36, 146.42, $146.49,147.02,147.08,147.22,147.30,147.55,147.63,149.26\left(\mathrm{~d}, J_{\text {C-P }}=8.4 \mathrm{~Hz}\right), 149.45\left(\mathrm{~d}, J_{\mathrm{C}-\mathrm{P}}=\right.$ $9.5 \mathrm{~Hz}), 152.13\left(\mathrm{~d}, J_{\mathrm{C}-\mathrm{P}}=5.4 \mathrm{H}\right), 152.15\left(\mathrm{~d}, J_{\mathrm{C}-\mathrm{P}}=6.0 \mathrm{~Hz}\right.$ ); HRMS (APCI-TOF, negative) $\mathrm{m} / \mathrm{z}$ calcd for $\mathrm{C}_{68} \mathrm{H}_{11} \mathrm{O}_{2} \mathrm{P}[\mathrm{M}-\mathrm{H}]^{-} 889.0418$, found: 889.0413.

\section{Synthesis}

6-[9-hydro $\left(C_{60}-I_{h}\right)[5,6]$ fulleren-1(9H)-yl]-dibenz[c,e][1,2]oxaphosphorin-6-oxide by the reaction of [60]fullerene and $6 \mathrm{H}$-dibenz$[c, e][1,2]$ oxaphosphorin-6-oxide (Table 1 , entry 10): To a solution of $\mathrm{C}_{60}(50.1 \mathrm{mg}, 69.6 \mu \mathrm{mol})$ in chlorobenzene $(20 \mathrm{~mL})$ was added DMSO (5 mL). To the solution was then added 6H-dibenz[c,e][1,2]oxaphosphorin-6-oxide (181 $\mathrm{mg}$, $836 \mu \mathrm{mol} ; 12.0$ equiv), and the mixture was stirred under air at $45^{\circ} \mathrm{C}$. HPLC analysis (Bucky-prep column, eluent: $i-\mathrm{PrOH} /$ toluene $=3 / 7$ ) of the mixture showed that yield of mono-adduct reached its maximum after 18 hours. The mixture was washed with brine, and the organic layer was dried over anhydrous $\mathrm{Na}_{2} \mathrm{SO}_{4}$. The crude solution was purified by silica gel column chromatography (5 g, eluent: chlorobenzene and 10\% ethyl acetate/toluene) to give $\mathrm{C}_{60}(4.00 \mathrm{mg}, 8 \%$ recovery) and the title compound $(24.2 \mathrm{mg}, 50 \%)$ as an analytically pure brown powder. IR (powder) 2883, 2849, 1549, 1475, 1447, 1428, 1258, 
1189, 1116, 941.8, 918.7, 889.4, 787.4, 752.7, $714.1 \mathrm{~cm}^{-1} ;{ }^{31} \mathrm{P}$ NMR (202.5 MHz, $\left.\mathrm{CDCl}_{3}\right) \delta 31.81$ (s); ${ }^{1} \mathrm{H}$ NMR (500 MHz, $\mathrm{CDCl}_{3}$ ) $\delta 8.44$ (ddd, $\left.J=6.3,1.2,22.1 \mathrm{~Hz}, 1 \mathrm{H}\right), 8.07$ (dd, $J=5.7,8.1$ $\mathrm{Hz}, 1 \mathrm{H}), 7.89(\mathrm{dd}, J=1.8,8.6 \mathrm{~Hz}, 1 \mathrm{H}), 7.84(\mathrm{t}, J=8.1 \mathrm{~Hz}, 1 \mathrm{H}), 7.64(\mathrm{td}, J=3.5,7.5 \mathrm{~Hz}, 1 \mathrm{H})$, $7.61(\mathrm{~d}, J=8.3 \mathrm{~Hz}, 1 \mathrm{H}), 7.503(\mathrm{~d}, J=27.5 \mathrm{~Hz}, 1 \mathrm{H}), 7.502(\mathrm{t}, J=7.5 \mathrm{~Hz}, 1 \mathrm{H}), 7.30(\mathrm{t}, J=7.8 \mathrm{~Hz}$, $1 \mathrm{H}) ;{ }^{13} \mathrm{C}$ NMR $\left(125 \mathrm{MHz}, \mathrm{CDCl}_{3}\right) \delta 55.45,69.80\left(\mathrm{~d}, J_{\mathrm{C}-\mathrm{P}}=98.8 \mathrm{~Hz}\right), 120.14\left(\mathrm{~d}, J_{\mathrm{C}-\mathrm{P}}=122.8 \mathrm{~Hz}\right)$, $120.29\left(\mathrm{~d}, J_{\mathrm{C}-\mathrm{P}}=7.2 \mathrm{~Hz}, \mathrm{CH}\right), 122.57\left(\mathrm{~d}, J_{\mathrm{C}-\mathrm{P}}=9.5 \mathrm{~Hz}\right), 124.06\left(\mathrm{~d}, J_{\mathrm{C}-\mathrm{P}}=10.7 \mathrm{~Hz}, \mathrm{CH}\right), 125.22$ $(\mathrm{CH}), 125.42(\mathrm{CH}), 128.75\left(\mathrm{~d}, J_{\mathrm{C}-\mathrm{P}}=13.1 \mathrm{~Hz}, \mathrm{CH}\right), 131.43(\mathrm{CH}), 133.05\left(\mathrm{~d}, J_{\mathrm{C}-\mathrm{P}}=9.0 \mathrm{~Hz}, \mathrm{CH}\right)$, $134.95\left(\mathrm{~d}, J_{\mathrm{C}-\mathrm{P}}=2.4 \mathrm{~Hz}, \mathrm{CH}\right), 135.28\left(\mathrm{~d}, J_{\mathrm{C}-\mathrm{P}}=2.4 \mathrm{~Hz}\right), 135.56\left(\mathrm{~d}, J_{\mathrm{C}-\mathrm{P}}=2.4 \mathrm{~Hz}\right), 136.26\left(\mathrm{~d}, J_{\mathrm{C}-\mathrm{P}}\right.$ $=5.4 \mathrm{~Hz}), 137.02\left(\mathrm{~d}, J_{\mathrm{C}-\mathrm{P}}=5.4 \mathrm{~Hz}\right), 138.08\left(\mathrm{~d}, J_{\mathrm{C}-\mathrm{P}}=6.6 \mathrm{~Hz}, \mathrm{CH}\right), 139.86\left(\mathrm{~d}, J_{\mathrm{C}-\mathrm{P}}=1.8 \mathrm{~Hz}\right)$, 140.50, 140.56, 140.97, 141.34, 141.48, 141.75, 141.79, 141.83, 141.86, 142.08, 142.12, 142.51, $142.52,142.71,142.74,143.17,144.21\left(\mathrm{~d}, J_{\mathrm{C}-\mathrm{P}}=1.2 \mathrm{~Hz}\right), 144.26\left(\mathrm{~d}, J_{\mathrm{C}-\mathrm{P}}=1.2 \mathrm{~Hz}\right), 144.68,144.73$, $145.29\left(\mathrm{~d}, J_{\mathrm{C}-\mathrm{P}}=1.8 \mathrm{~Hz}\right), 145.40,145.43,145.51,145.55,145.65,145.69,145.92,146.30,146.32$, $146.33,146.40,146.42,146.52\left(\mathrm{~d}, J_{\mathrm{C}-\mathrm{P}}=1.8 \mathrm{~Hz}\right), 146.57,146.93,147.23\left(\mathrm{~d}, J_{\mathrm{C}-\mathrm{P}}=8.4 \mathrm{~Hz}\right)$, $147.33\left(\mathrm{~d}, J_{\mathrm{C}-\mathrm{P}}=1.8 \mathrm{~Hz}\right), 147.55\left(\mathrm{~d}, J_{\mathrm{C}-\mathrm{P}}=7.2 \mathrm{~Hz}\right), 147.64\left(\mathrm{~d}, J_{\mathrm{C}-\mathrm{P}}=8.4 \mathrm{~Hz}\right), 151.42\left(\mathrm{~d}, J_{\mathrm{C}-\mathrm{P}}=8.9\right.$ $\mathrm{Hz}), 151.71\left(\mathrm{~d}, J_{\mathrm{C}-\mathrm{P}}=5.4 \mathrm{~Hz}\right), 151.87\left(\mathrm{~d}, J_{\mathrm{C}-\mathrm{P}}=4.8 \mathrm{~Hz}\right.$ ); HRMS (APCI-TOF, negative) $\mathrm{m} / \mathrm{z}$ calcd for $\mathrm{C}_{72} \mathrm{H}_{9} \mathrm{O}_{2} \mathrm{P}[\mathrm{M}-\mathrm{H}]^{-}$935.0262, found: 935.0261.

Synthesis of dimethyl[9-hydro $\left(C_{60}-I_{h}\right)[5,6]$ fulleren-1 $(9 H)$-yl]phosphonate by the reaction of [60]fullerene and dimethylphosphite (Table 1, entry 11): To a solution of $\mathrm{C}_{60}$ (50.3 mg, $69.8 \mu \mathrm{mol})$ in chlorobenzene $(20 \mathrm{~mL})$ was added HMPA $(5 \mathrm{~mL})$. To the solution was then added dimethylphosphite (154 mg, $1.40 \mathrm{mmol} ; 20$ equiv), and the mixture was stirred under air at $120^{\circ} \mathrm{C}$. HPLC analysis (Bucky-prep column, eluent: $i-\mathrm{PrOH} /$ toluene $=3 / 7$ ) of the mixture showed that yield of mono-adduct reached its maximum after 8 hours. The mixture was washed with brine, and the organic layer was dried over anhydrous $\mathrm{Na}_{2} \mathrm{SO}_{4}$. The crude solution was purified by silica gel column chromatography (5 g, eluent: chlorobenzene and $10 \%$ ethyl acetate/toluene) to give $\mathrm{C}_{60}(12.1 \mathrm{mg}, 24 \%$ recovery) and the title compound $(23.3 \mathrm{mg}, 40 \%)$ as an analytically pure brown powder. The product was identical with an authentic sample by HPLC, IR, NMR and MS analyses. ${ }^{1}$

\section{Synthesis}

of

2-[9-hydro $\left(\mathrm{C}_{60}-\mathrm{I}_{\mathrm{h}}\right)[5,6]$ fulleren-1(9H)-yl]-5,5-dimethyl-1,3,2-dioxaphosphorinan-2-one by the reaction of [60]fullerene and 5,5-dimethyl-1,3,2-dioxaphosphorinan-2-one (Table 1, entry 12): To a solution of $\mathrm{C}_{60}(33.7 \mathrm{mg}, 46.7 \mu \mathrm{mol})$ in chlorobenzene $(20 \mathrm{~mL})$ was added DMSO $(5 \mathrm{~mL})$. To the solution was then added 5,5-dimethyl-1,3,2-dioxaphosphorinan-2-one (140 mg, $934 \mu \mathrm{mol} ; 20$ equiv), and the mixture was stirred under air at $120{ }^{\circ} \mathrm{C}$. HPLC analysis (Bucky-prep column, eluent: S6 
$i$-PrOH/toluene $=3 / 7)$ of the mixture showed that yield of mono-adduct reached its maximum after 30 hours. The mixture was washed with brine, and the organic layer was dried over anhydrous $\mathrm{Na}_{2} \mathrm{SO}_{4}$. The crude solution was purified by silica gel column chromatography (5 g, eluent: chlorobenzene and 10\% ethyl acetate/toluene) to give $\mathrm{C}_{60}$ (7.90 $\mathrm{mg}, 23 \%$ recovery) and the title compound $(17.65 \mathrm{mg}, 43 \%)$ as an analytically pure brown powder. IR (powder) 2957, 2930, 2887, 1463, 1424, 1266, 1208, 1181, 1058, 1015, 995.9, 945.7, 903.2, 822.2, 799.9, 748.8, $729.5 \mathrm{~cm}^{-1}$; ${ }^{31} \mathrm{P}$ NMR (202.5 MHz, $\left.\mathrm{CDCl}_{3}\right) \delta 15.48$ (s); ${ }^{1} \mathrm{H}$ NMR (500 MHz, CDCl $)$ ) $7.37(\mathrm{~d}, J=29.8 \mathrm{~Hz}, 1 \mathrm{H}), 4.68(\mathrm{dd}, J=8.0,10.9 \mathrm{~Hz}, 2 \mathrm{H}), 4.40(\mathrm{dd}$,

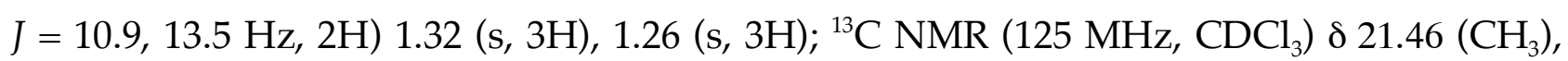
$21.73\left(\mathrm{CH}_{3}\right), 33.45\left(\mathrm{~d}, J_{\mathrm{C}-\mathrm{P}}=6.6 \mathrm{~Hz}\right), 56.30\left(\mathrm{~d}, J_{\mathrm{C}-\mathrm{P}}=1.7 \mathrm{~Hz}\right), 65.17\left(\mathrm{~d}, J_{\mathrm{C}-\mathrm{P}}=148.4 \mathrm{~Hz}\right), 77.95$ $\left(\mathrm{d}, J_{\mathrm{C}-\mathrm{P}}=7.2 \mathrm{~Hz}, \mathrm{OCH}_{2}\right), 135.52\left(\mathrm{~d}, J_{\mathrm{C}-\mathrm{P}}=2.4 \mathrm{~Hz}\right), 136.62\left(\mathrm{~d}, J_{\mathrm{C}-\mathrm{P}}=6.0 \mathrm{~Hz}\right), 140.31\left(\mathrm{~d}, J_{\mathrm{C}-\mathrm{P}}=1.2\right.$ Hz), 140.68, 141.57, 141.60, 141.63, 141.83, 142.00, 142.04, 142.14, 142.66, 142.76, 143.33, $144.38,144.40,144.79,145.49,145.54,145.67,145.69,145.93,146.37,146.42,146.45,146.58$, $147.06,147.25\left(\mathrm{~d}, J_{\mathrm{C}-\mathrm{P}}=2.4 \mathrm{~Hz}\right), 147.35\left(\mathrm{~d}, J_{\mathrm{C}-\mathrm{P}}=1.8 \mathrm{~Hz}\right), 148.22,148.32,151.63\left(\mathrm{~d}, J_{\mathrm{C}-\mathrm{P}}=6.6\right.$ $\mathrm{Hz}$ ); MS (ESI-TOF, negative) $\mathrm{m} / \mathrm{z}$ calcd for $\mathrm{C}_{65} \mathrm{H}_{11} \mathrm{O}_{3} \mathrm{P}[\mathrm{M}-\mathrm{H}]^{-}$869.0367, found: 869.0362. 
NMR and mass spectra

${ }^{31}$ P NMR spectrum (Table 1 , entry 5 )

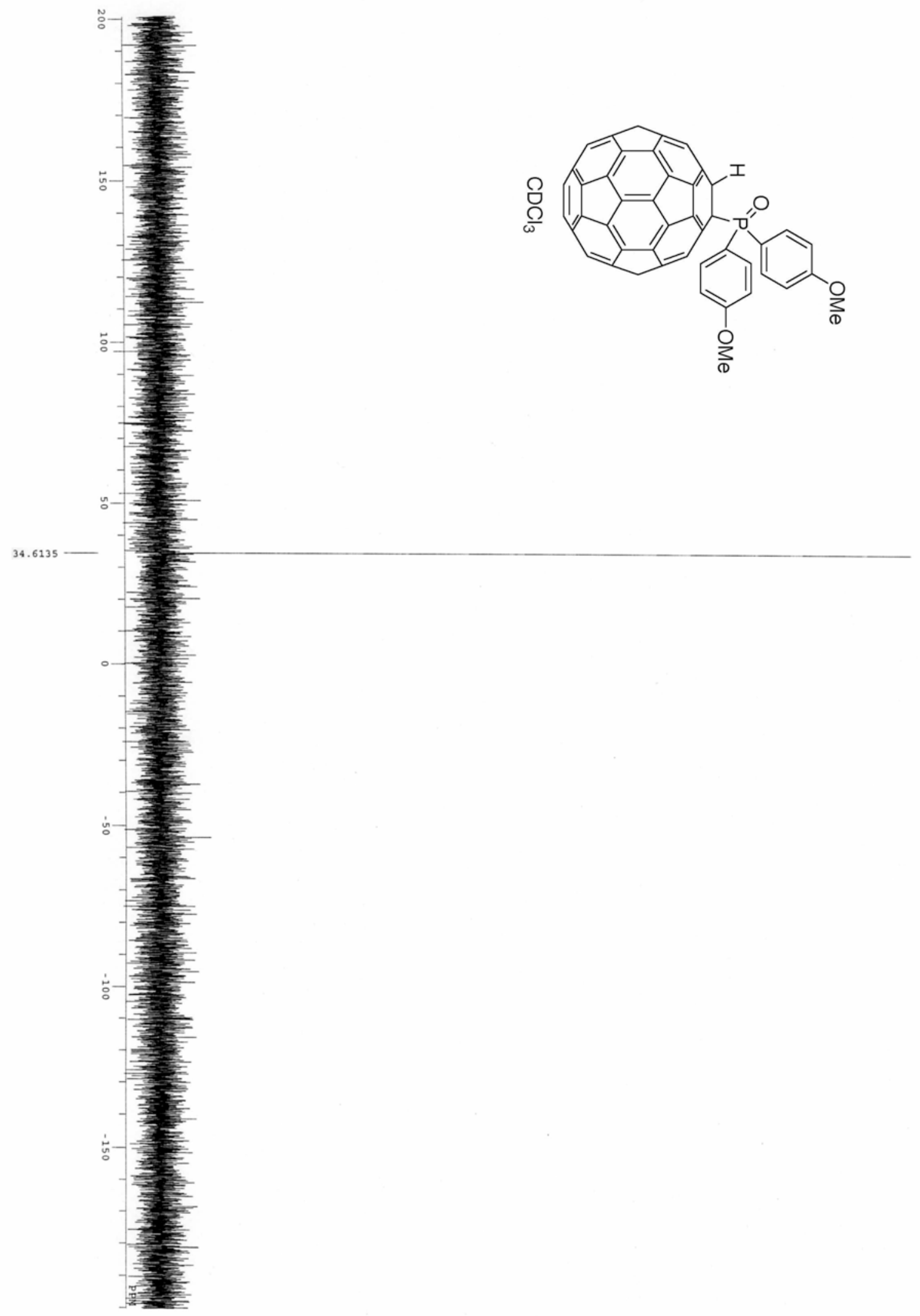


${ }^{1} \mathrm{H}$ NMR spectrum (Table 1, entry 5)

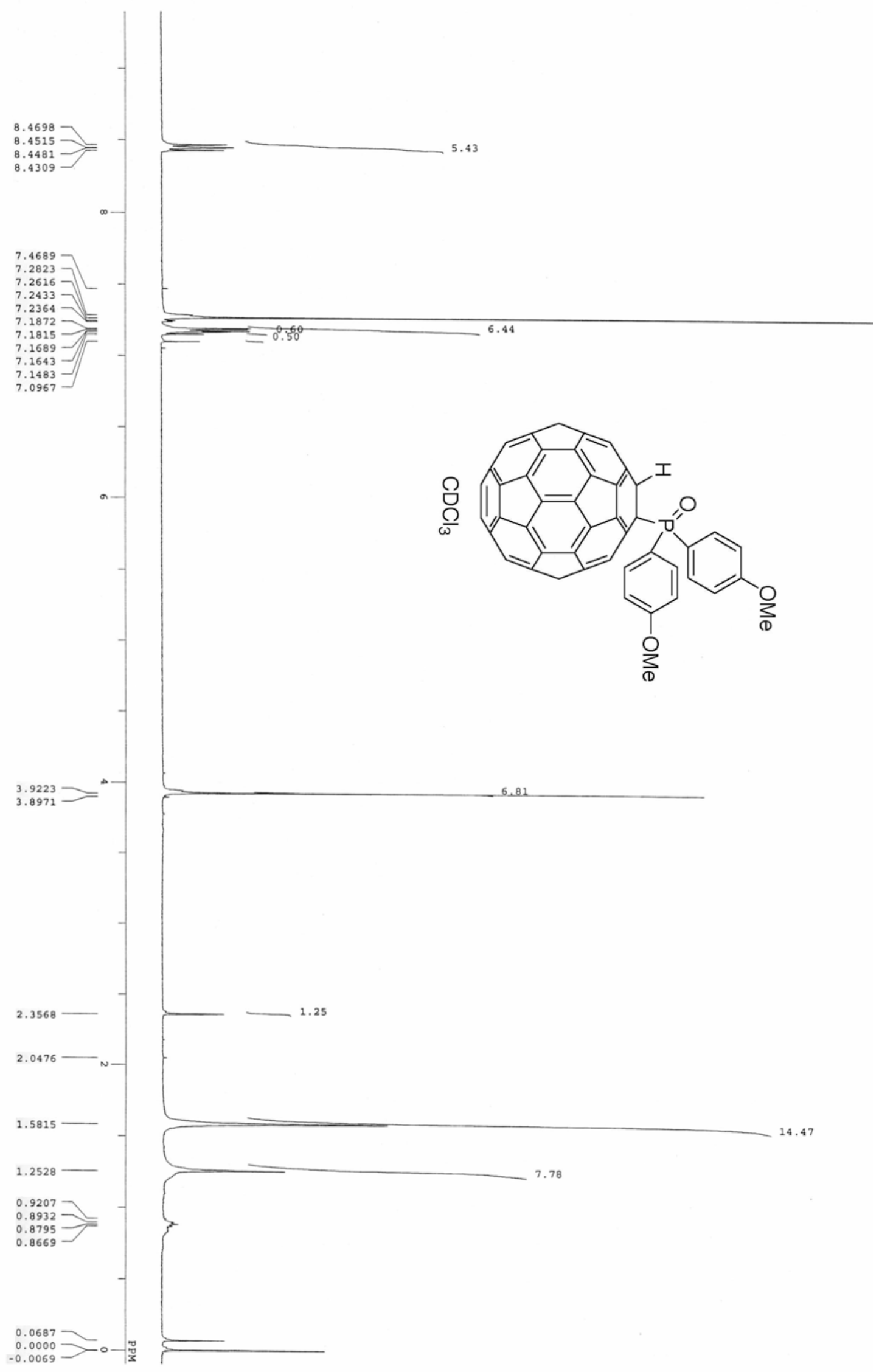


${ }^{13} \mathrm{C}$ NMR spectrum (Table 1, entry 5)

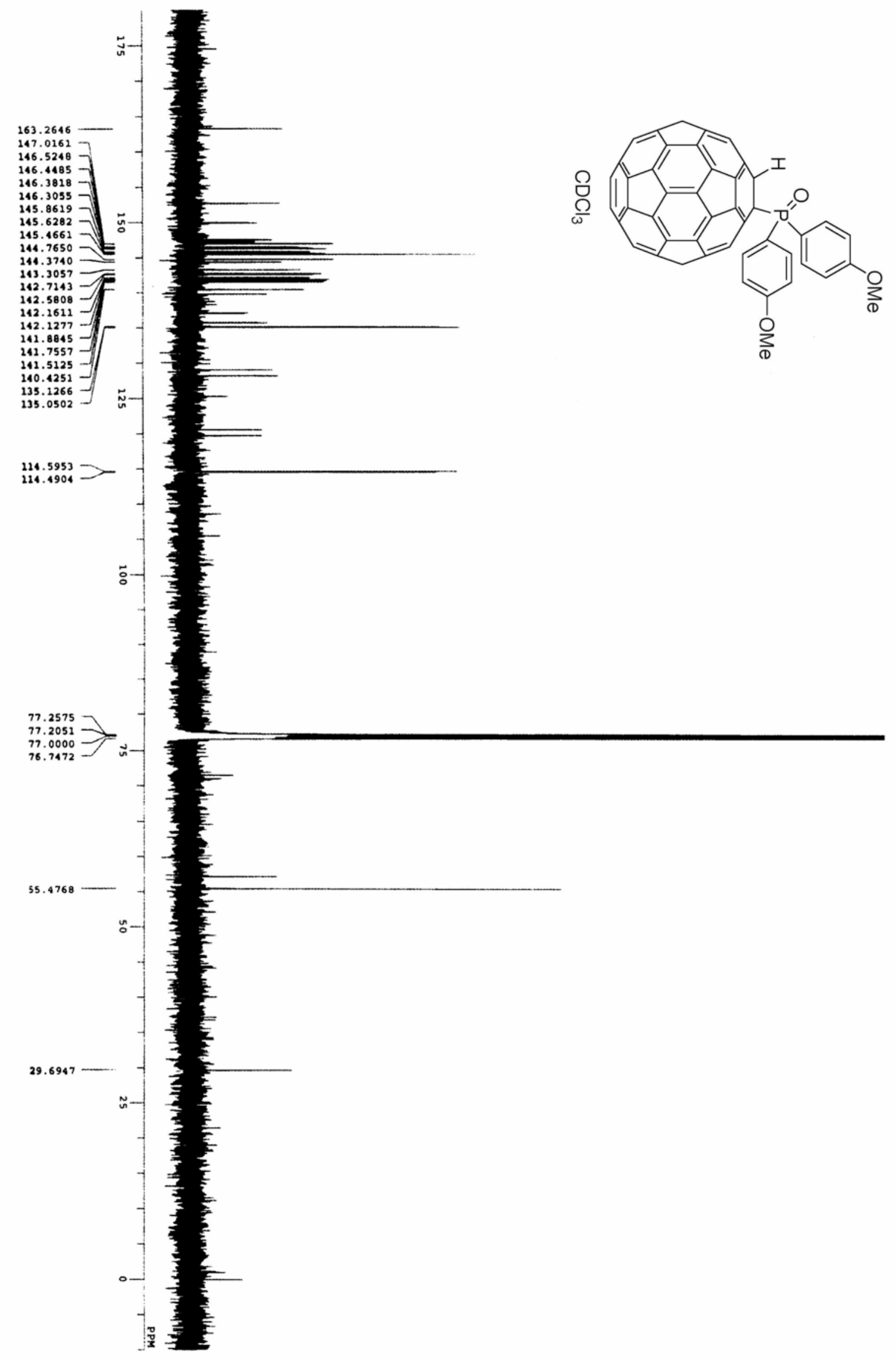


DEPT (135) spectrum (Table 1, entry 5)

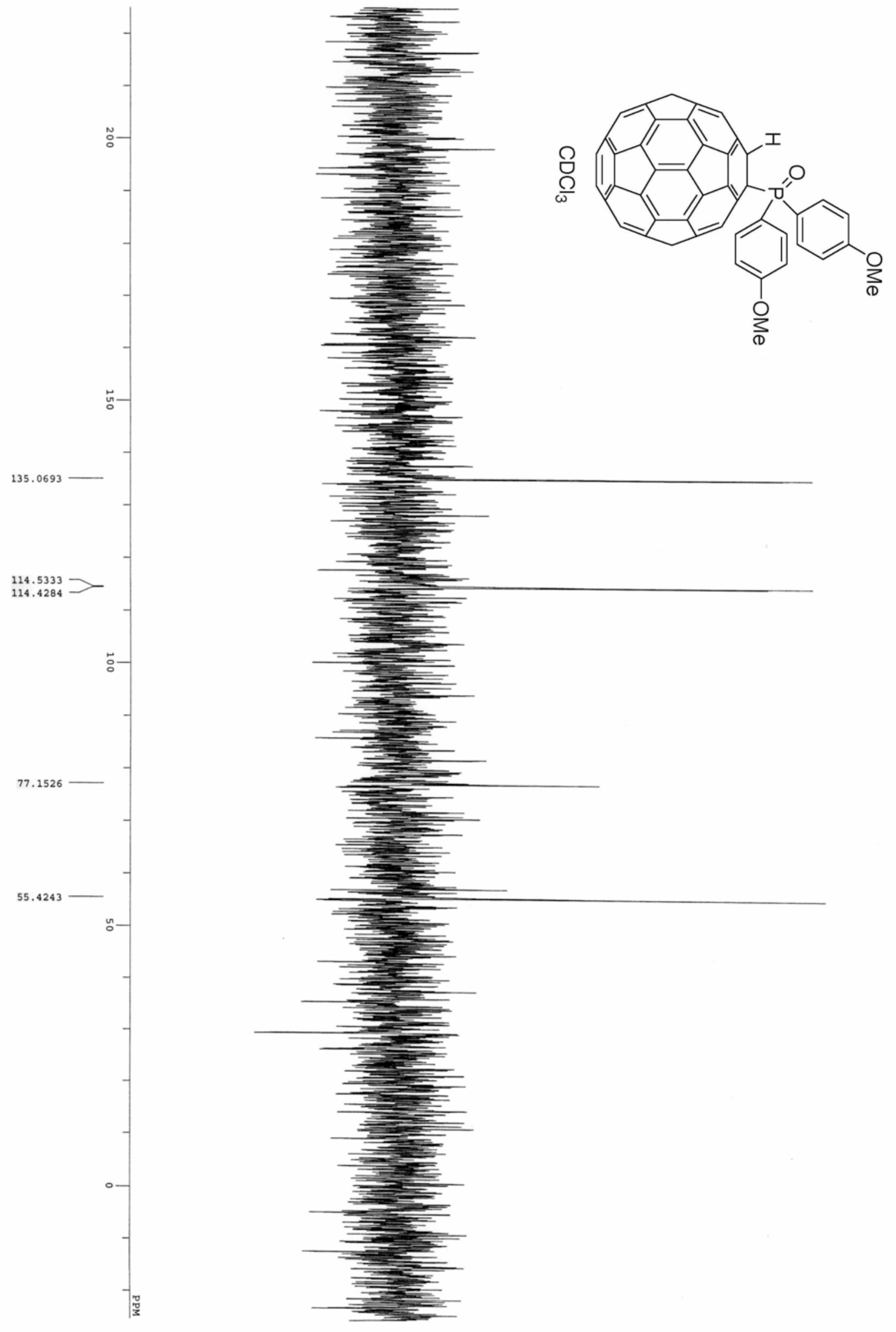


MS spectrum (Table 1, entry 5)

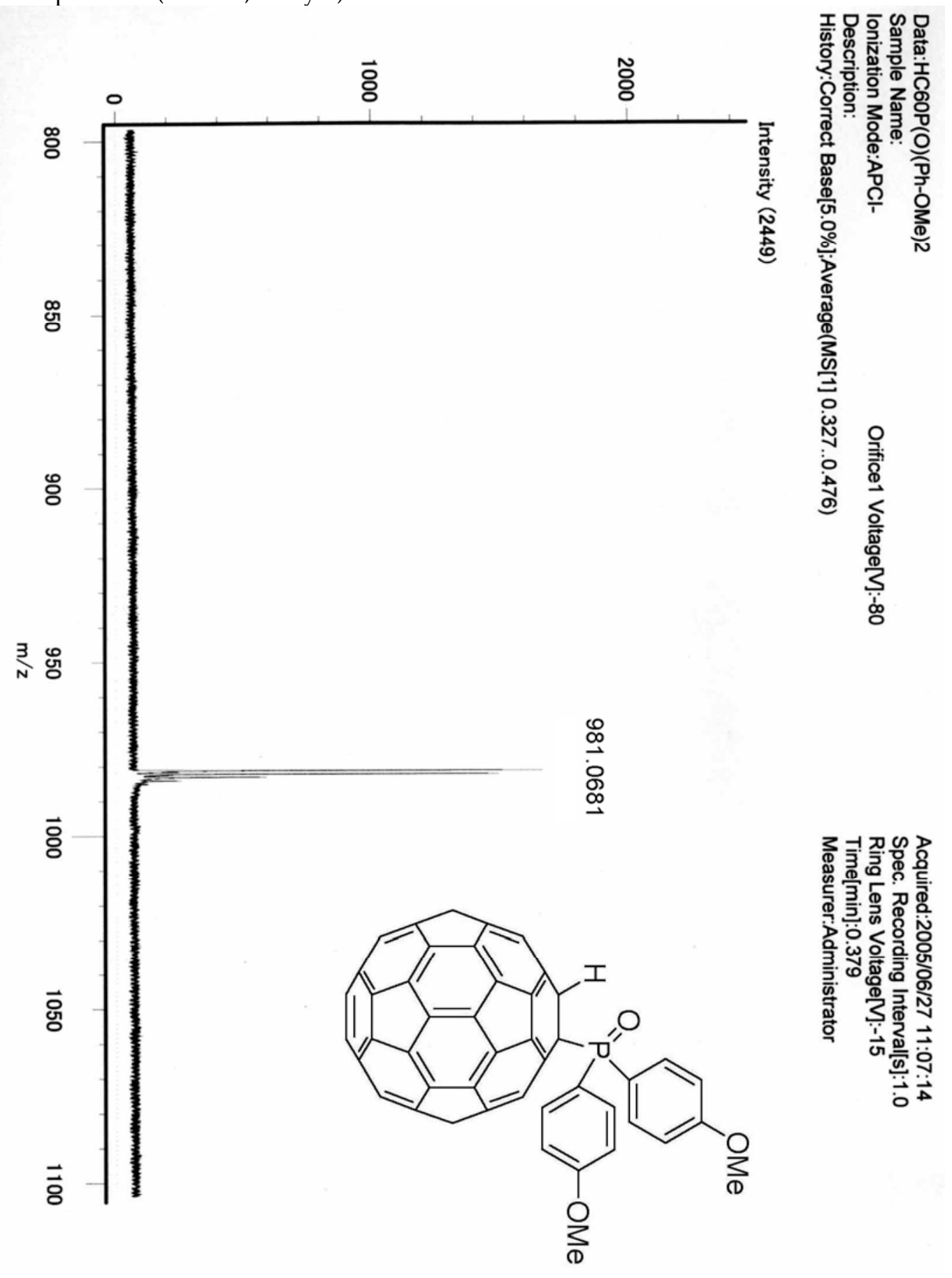


${ }^{31}$ P NMR spectrum (Table 1, entry 6)

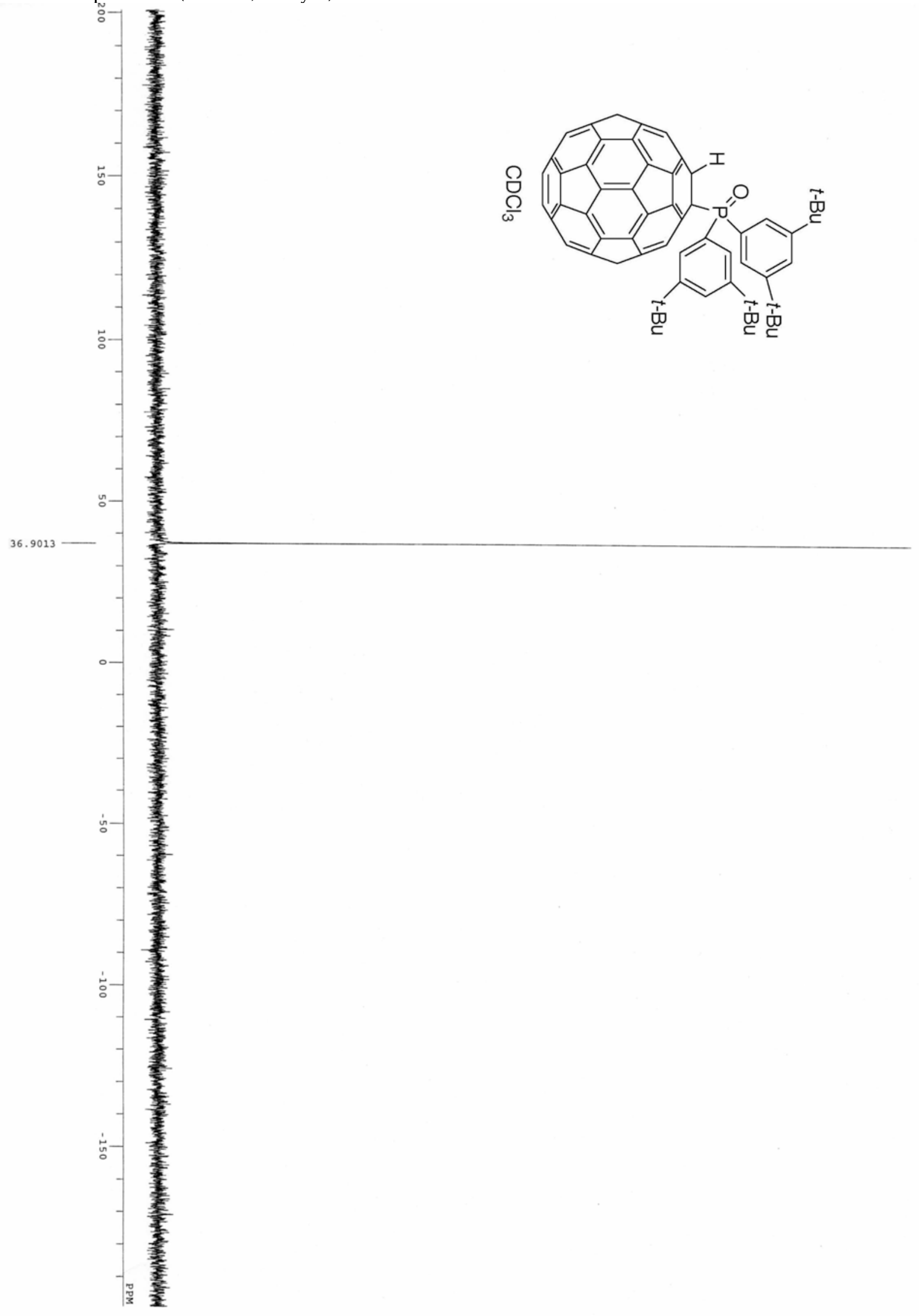


${ }^{1} \mathrm{H}$ NMR spectrum (Table 1, entry 6)

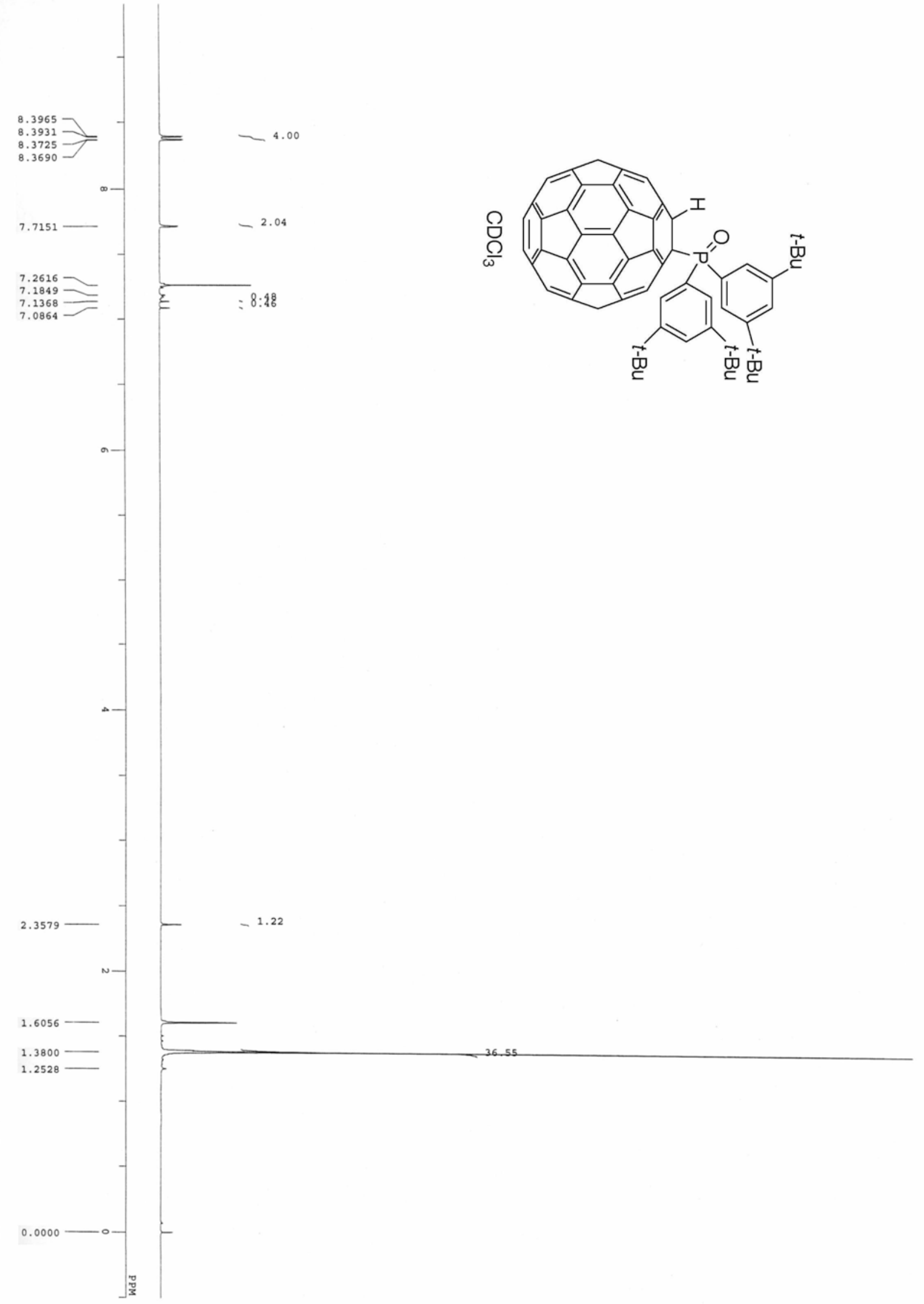


${ }^{13} \mathrm{C}$ NMR spectrum (Table 1, entry 6)

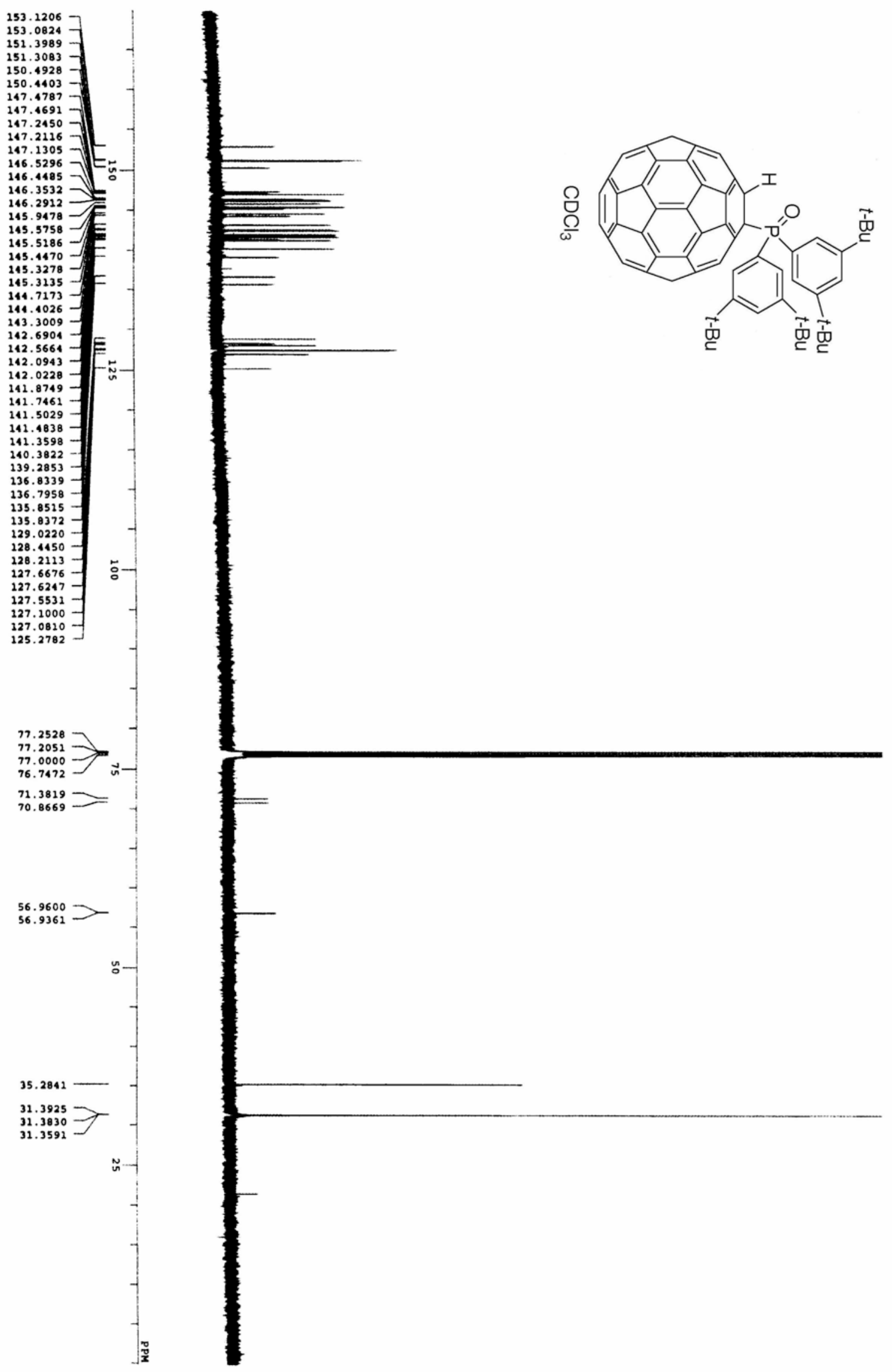


DEPT $\left(135^{\circ}\right)$ spectrum (Table 1, entry 6)

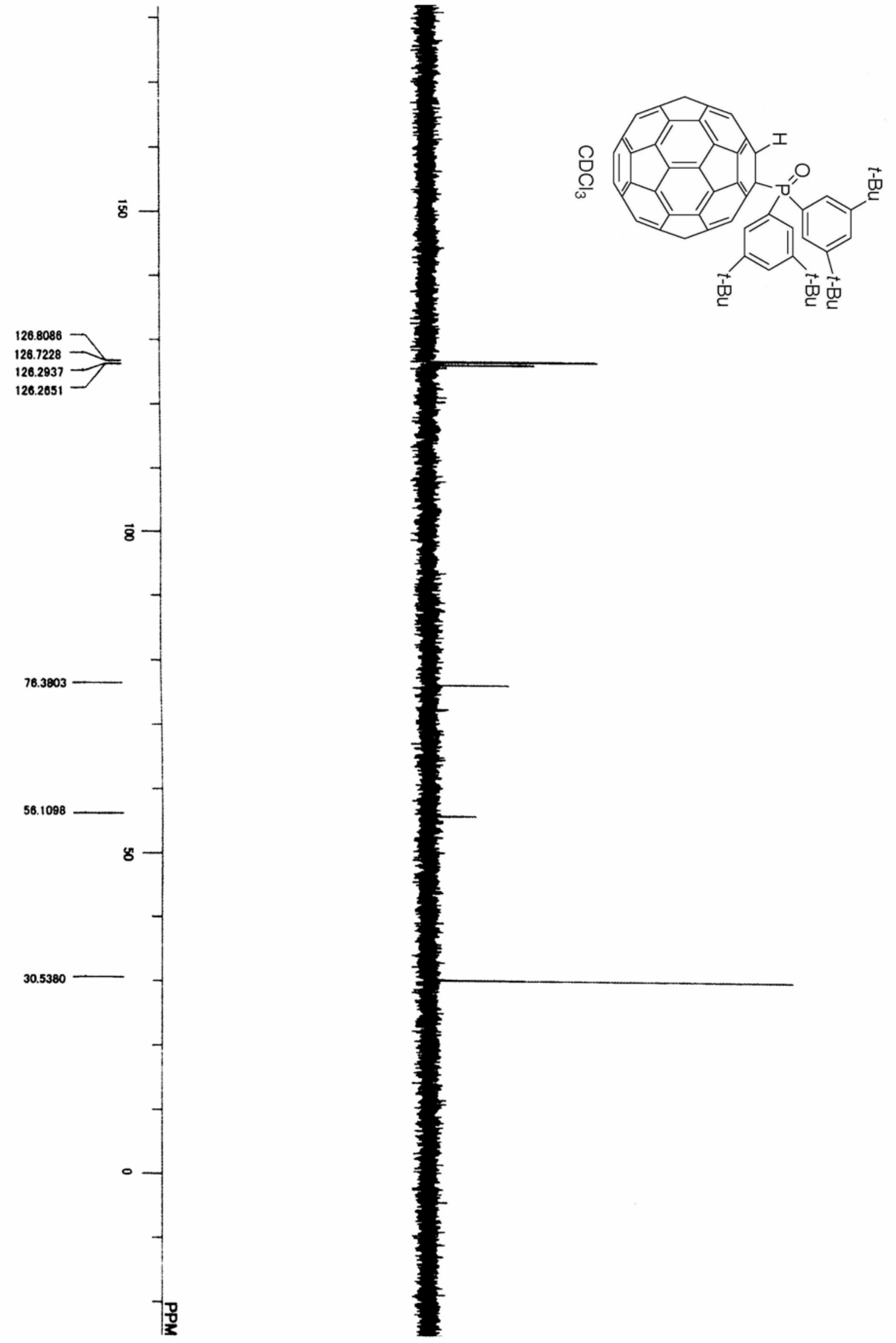


MS spectrum (Table 1, entry 6)

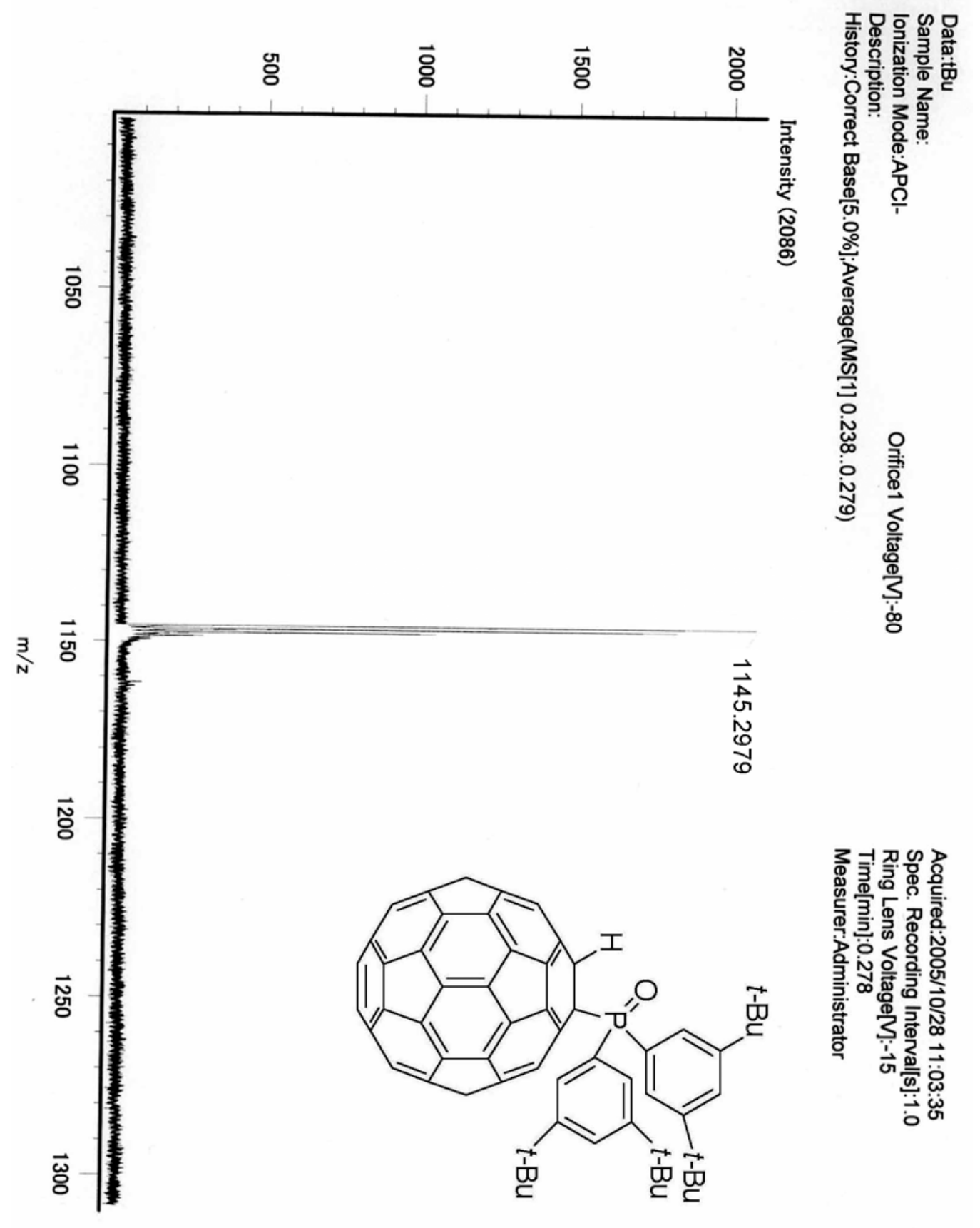


${ }^{31}$ P NMR spectrum (Table 1, entry 7)

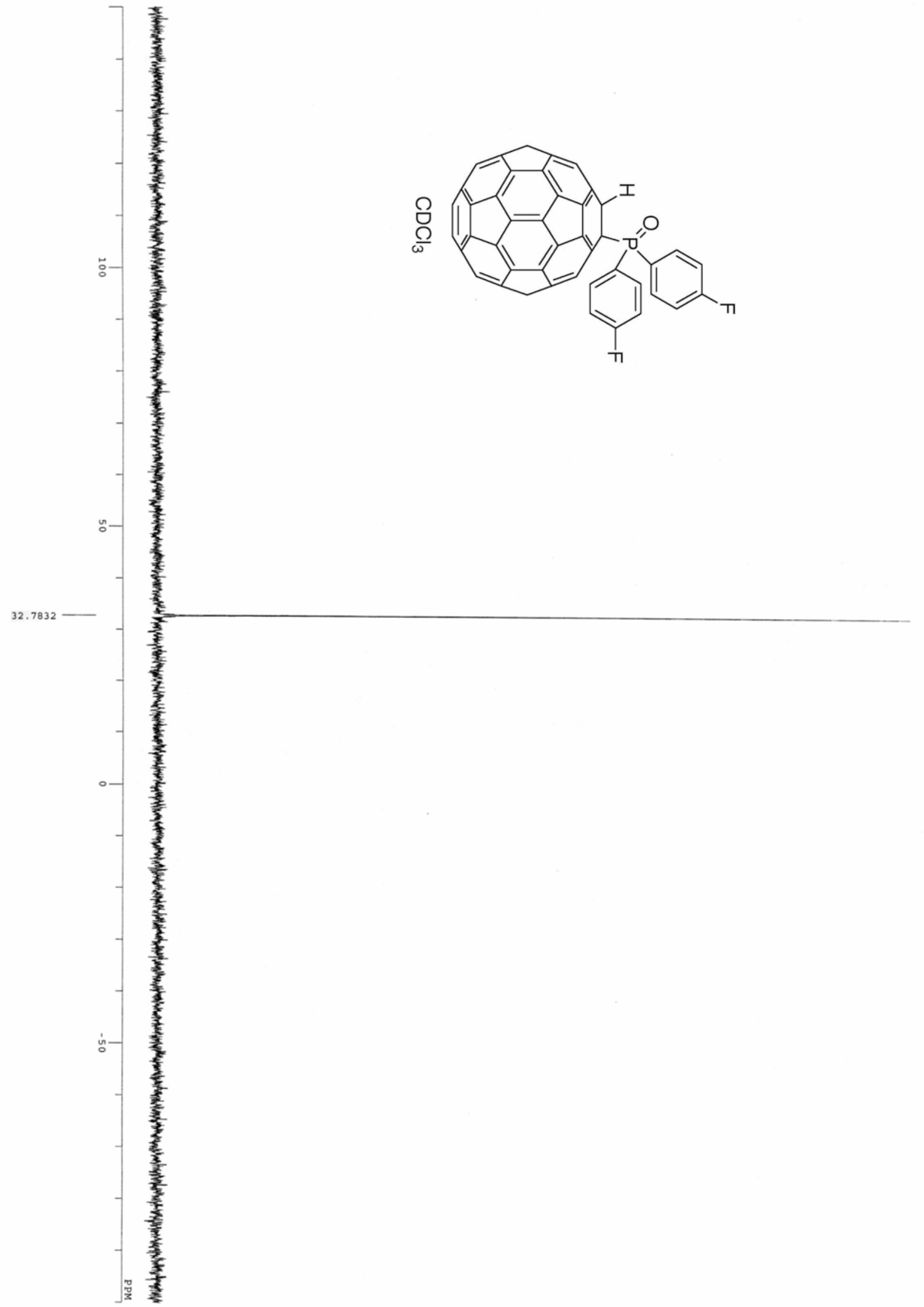


${ }^{1} \mathrm{H}$ NMR spectrum (Table 1, entry 7 )

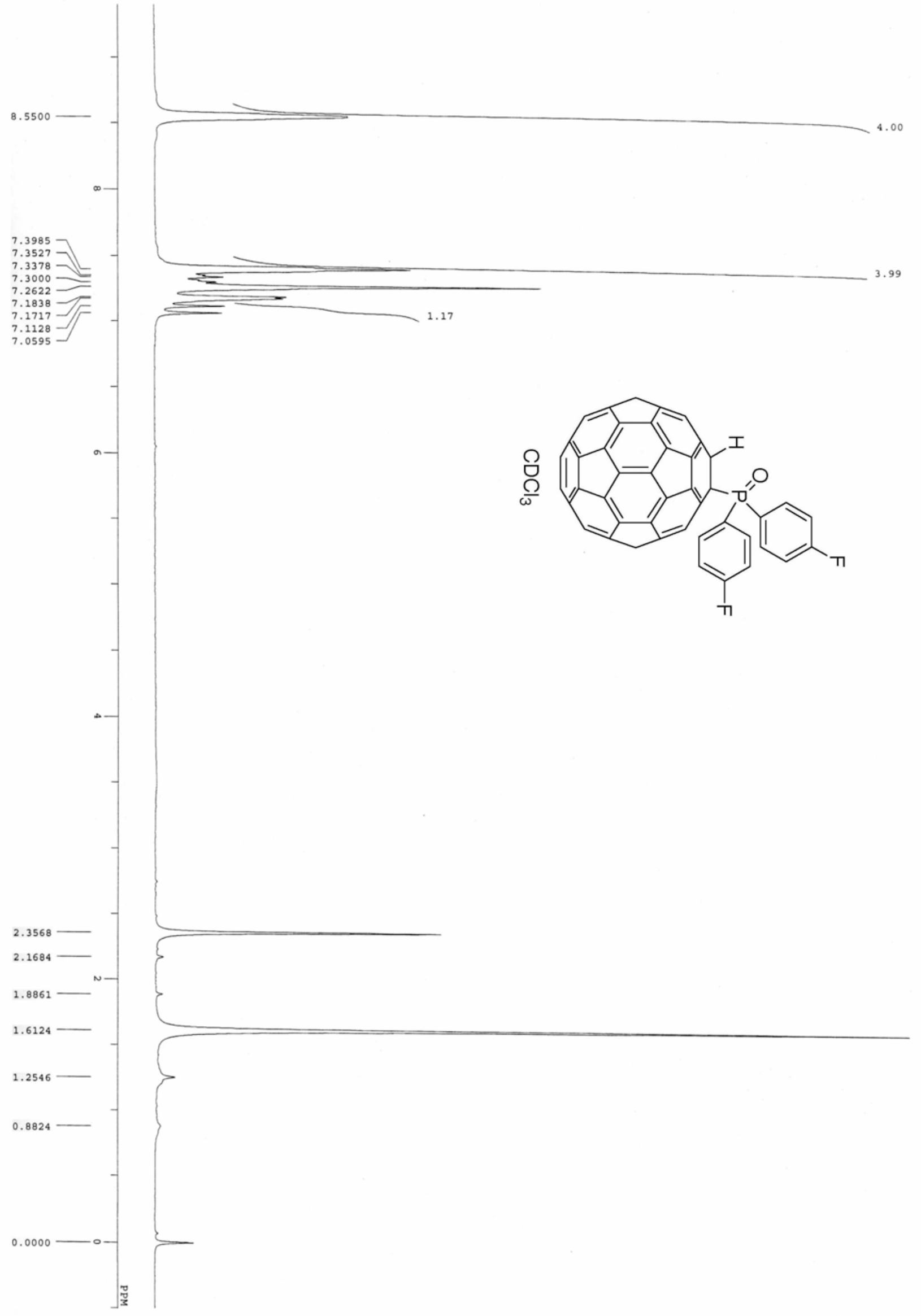


${ }^{13} \mathrm{C}$ NMR spectrum (Table 1, entry 7)

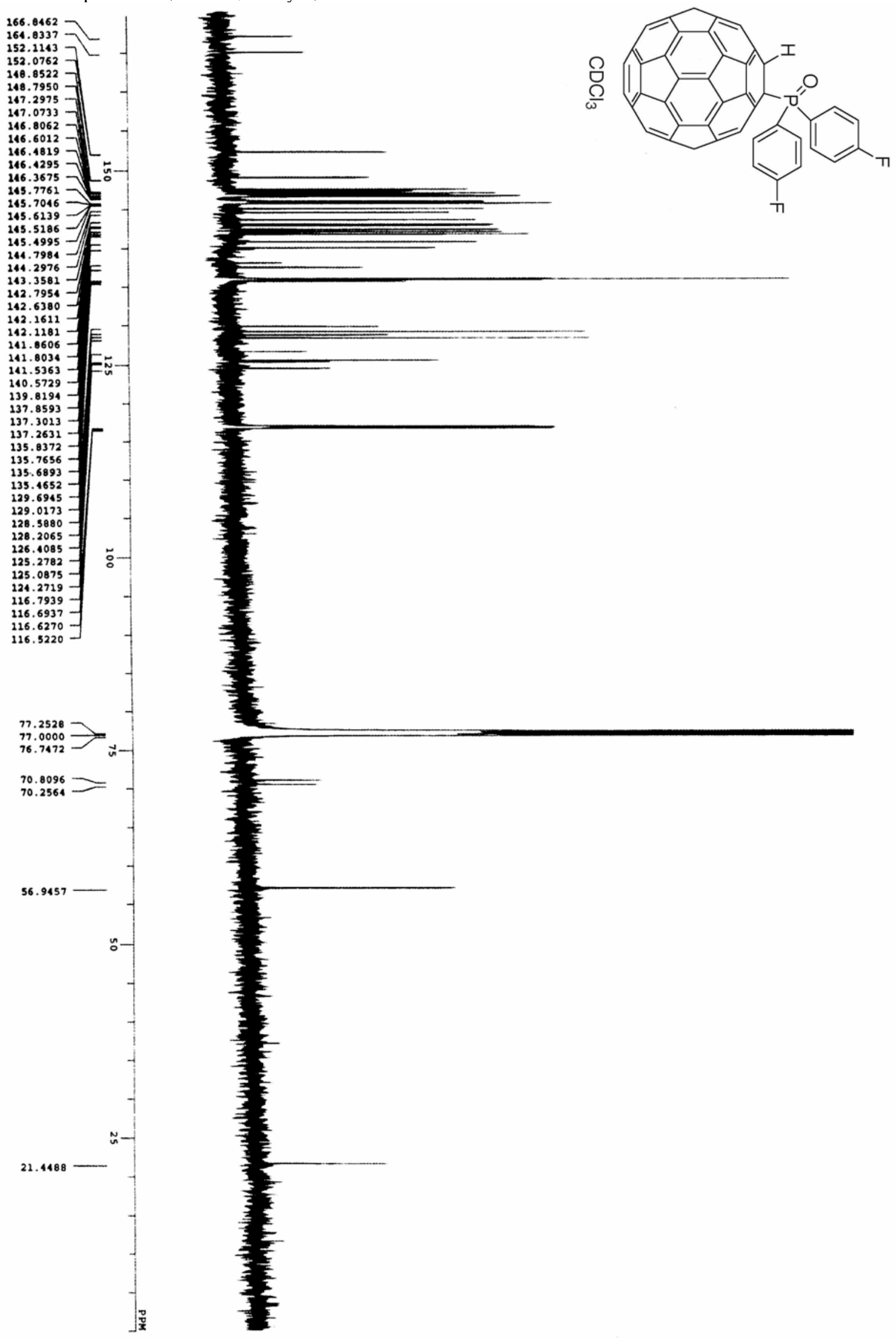


MS spectrum (Table 1, entry 7)

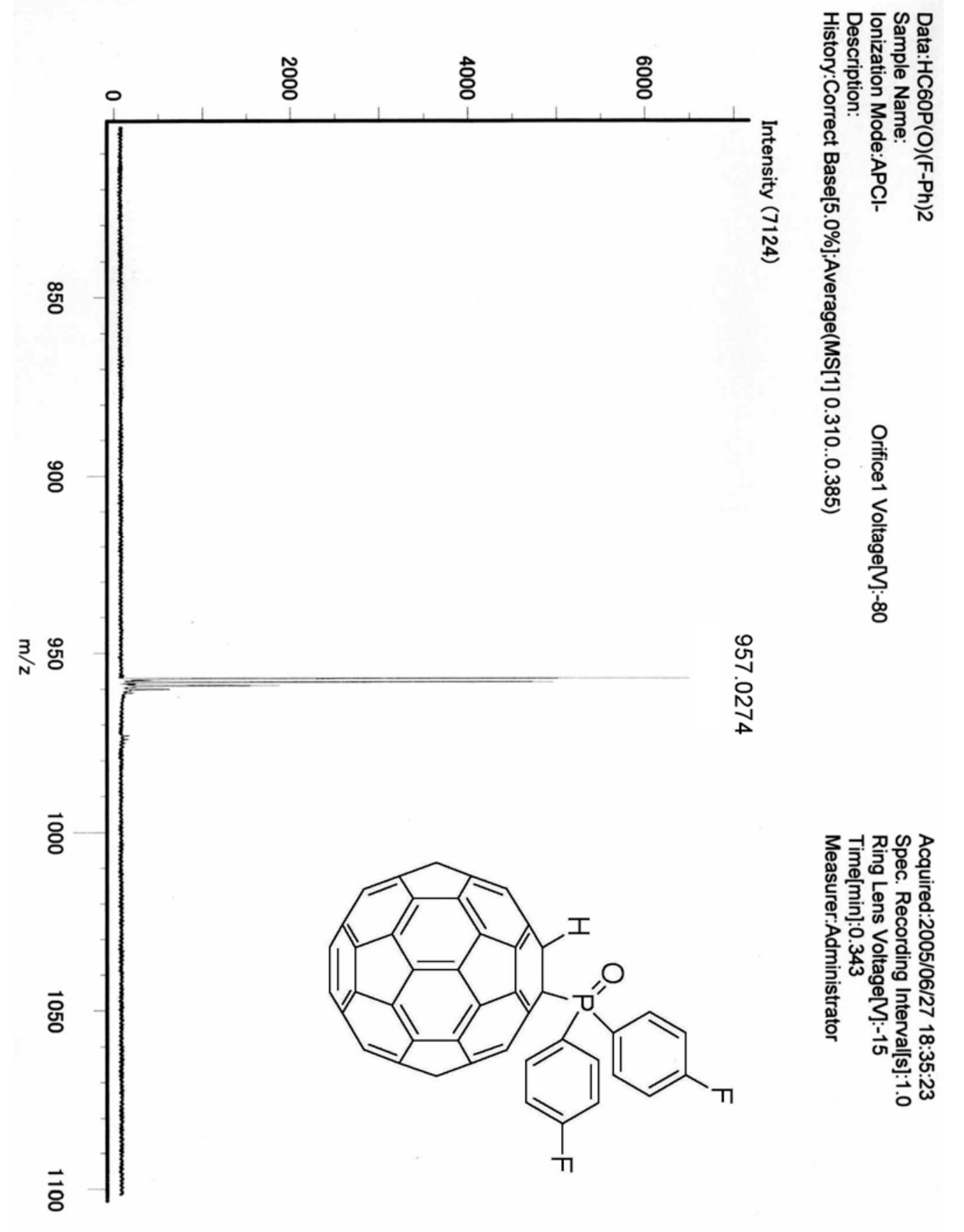


${ }^{31}$ P NMR spectrum (Table 1, entry 8 )

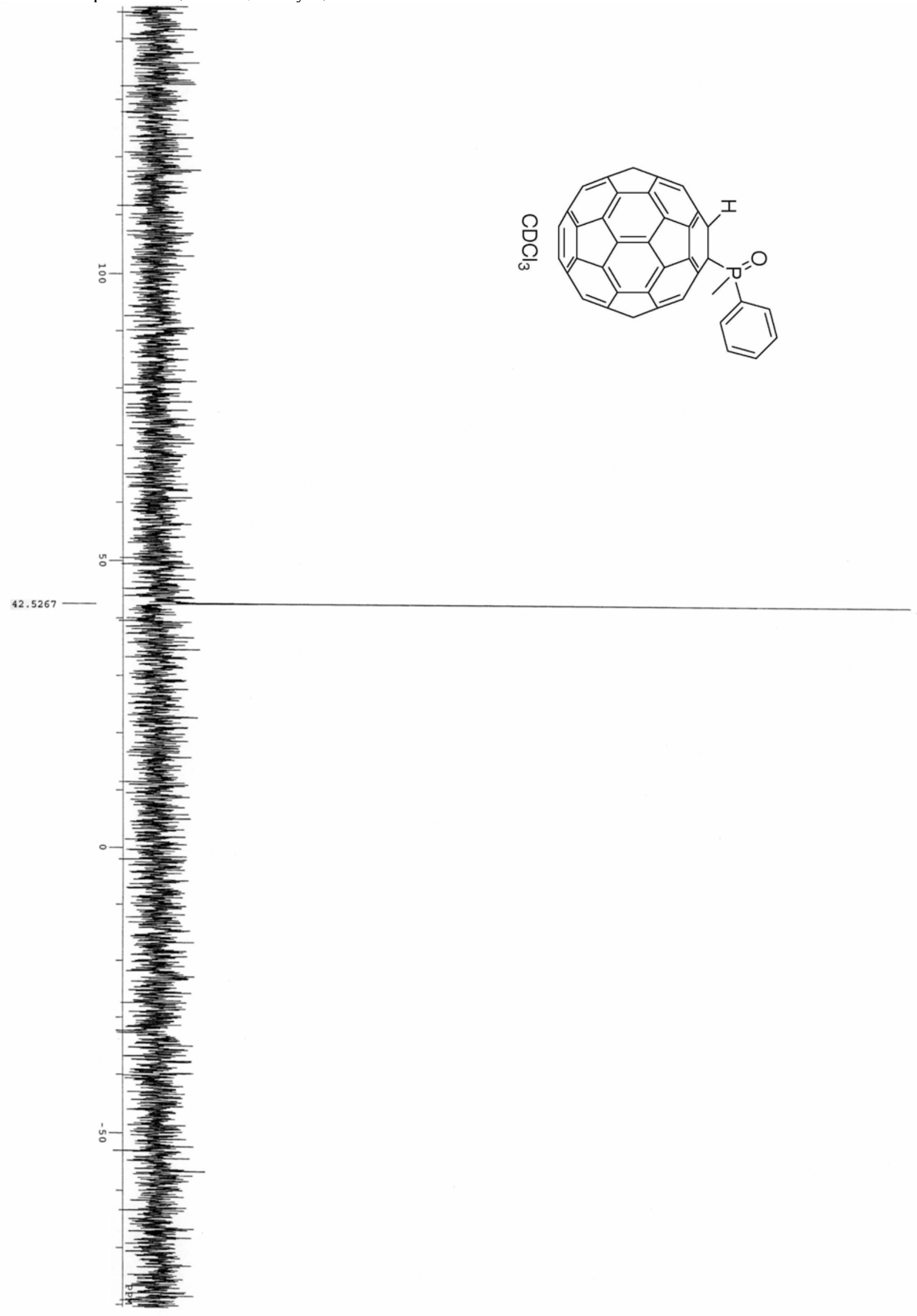


${ }^{1} \mathrm{H}$ NMR spectrum (Table 1, entry 8 )

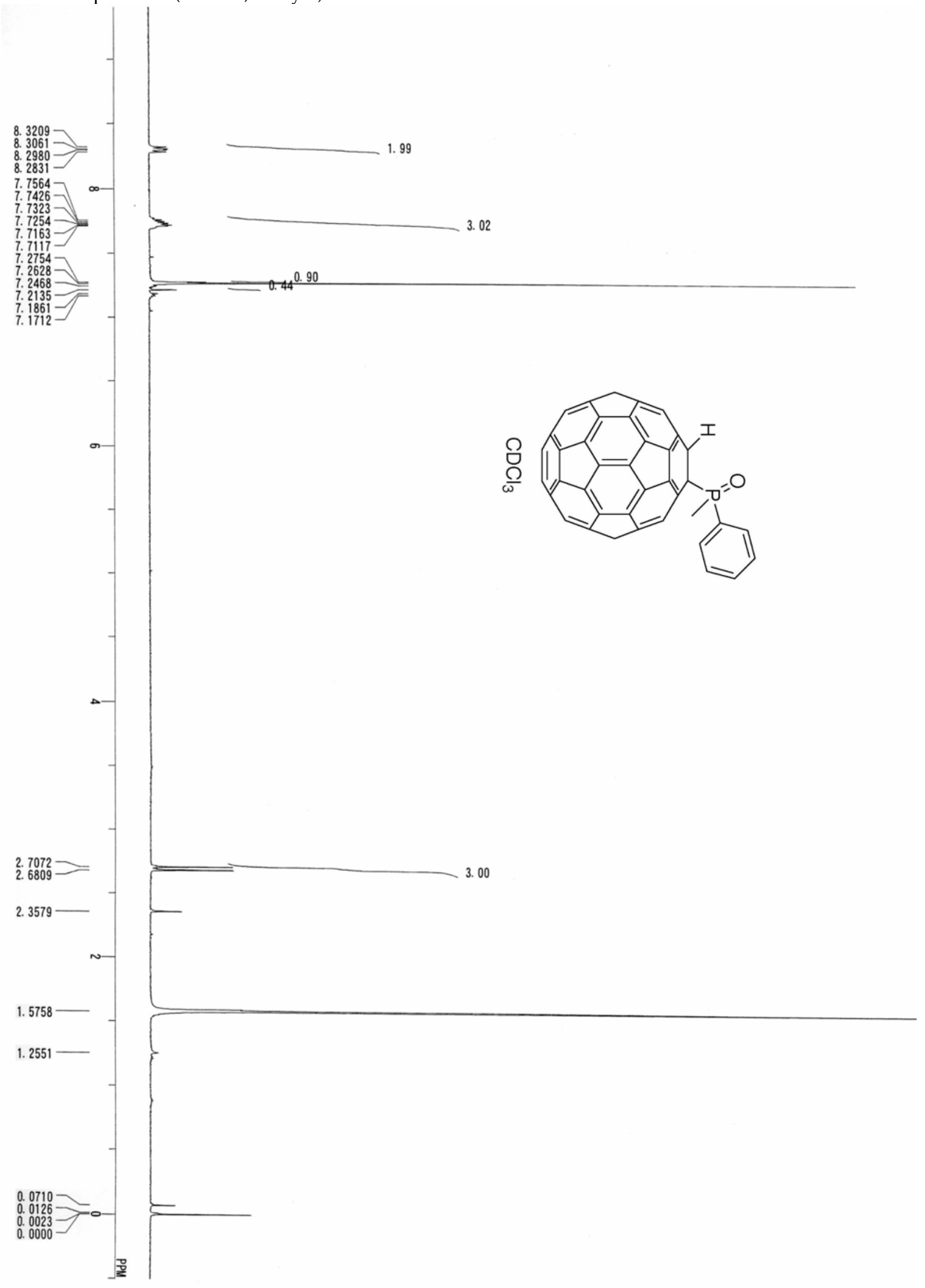


${ }^{13} \mathrm{C}$ NMR spectrum (Table 1, entry 8)

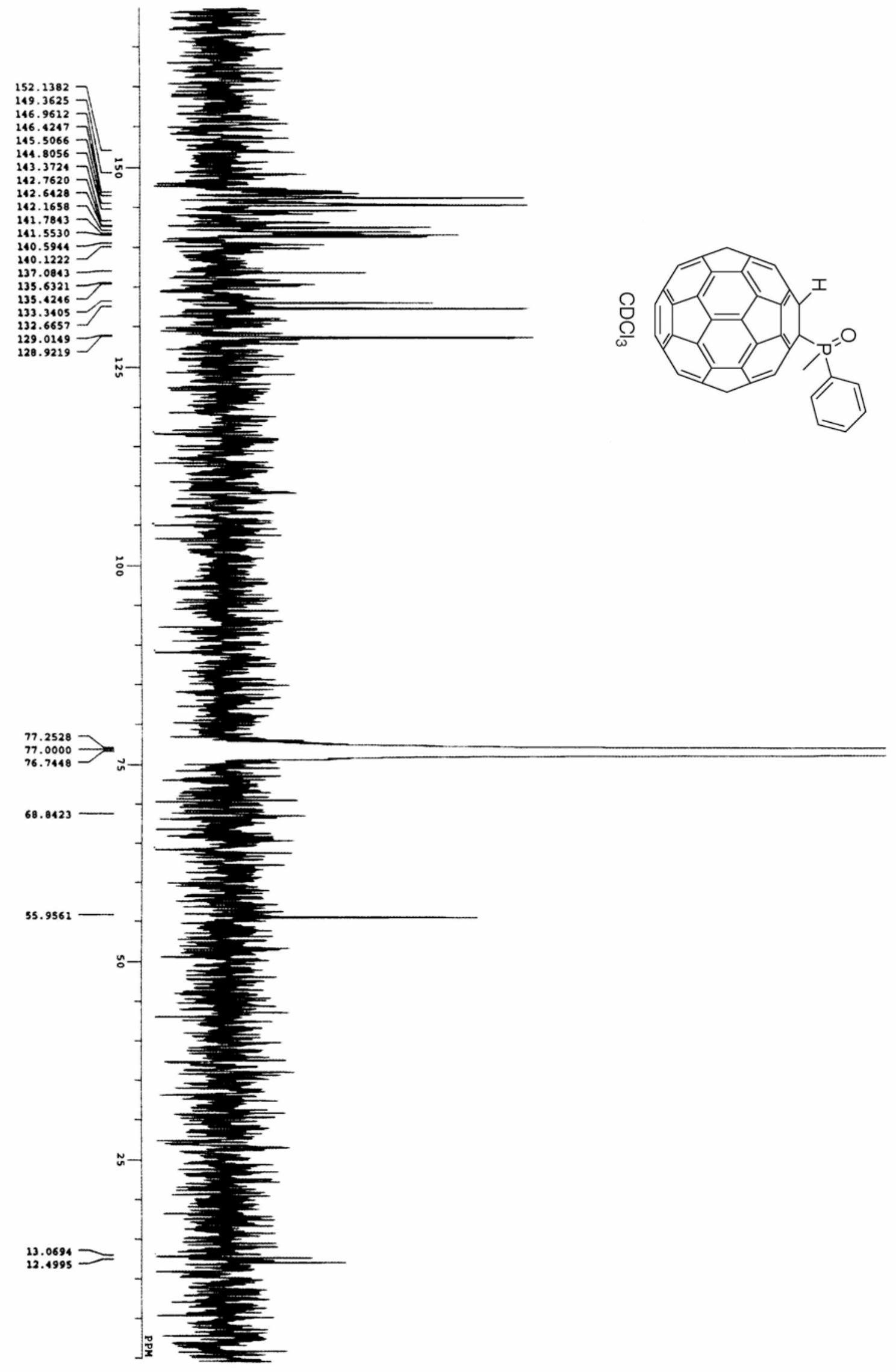


MS spectrum (Table 1, entry 8 )

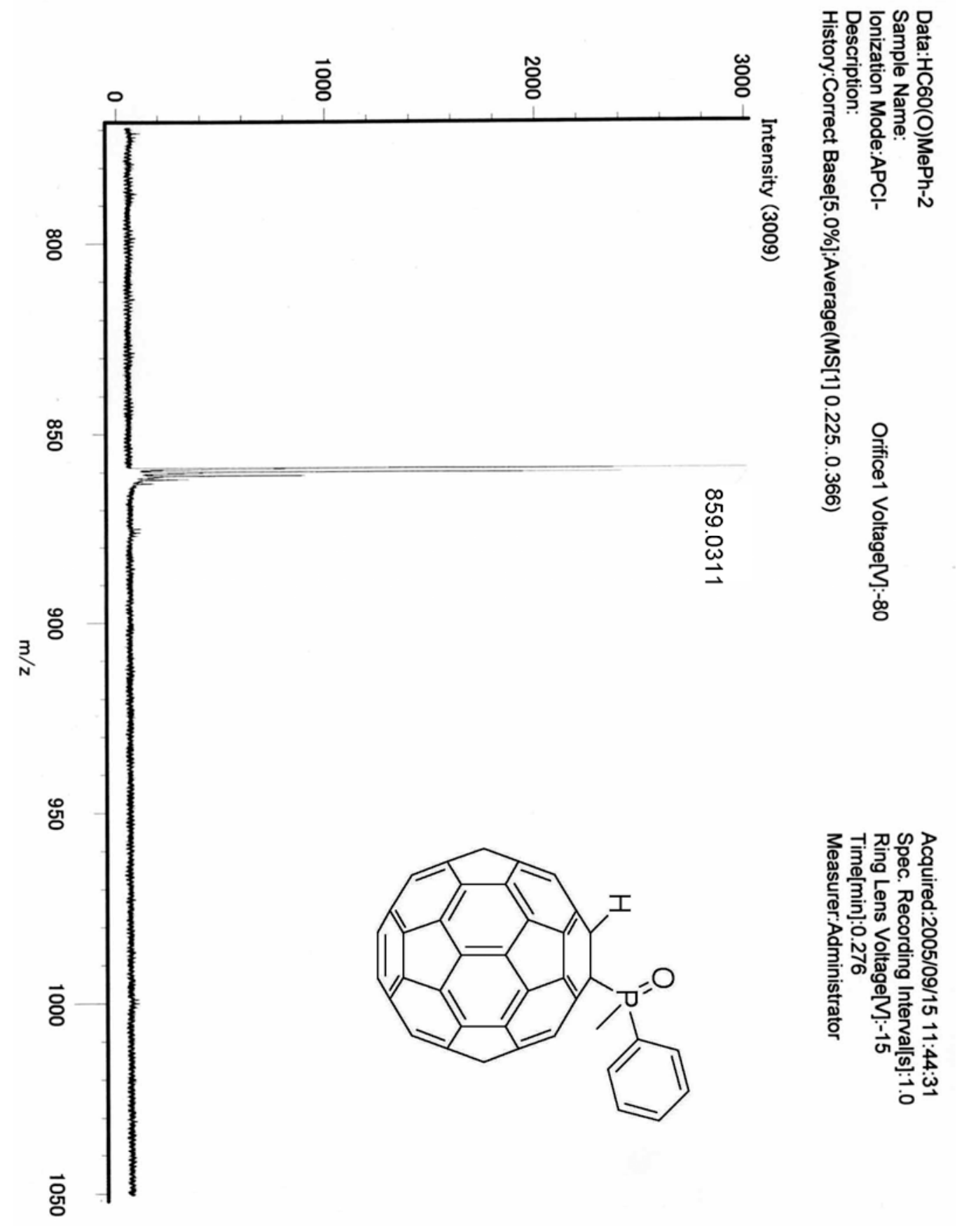


${ }^{31}$ P NMR spectrum (Table 1, entry 9)

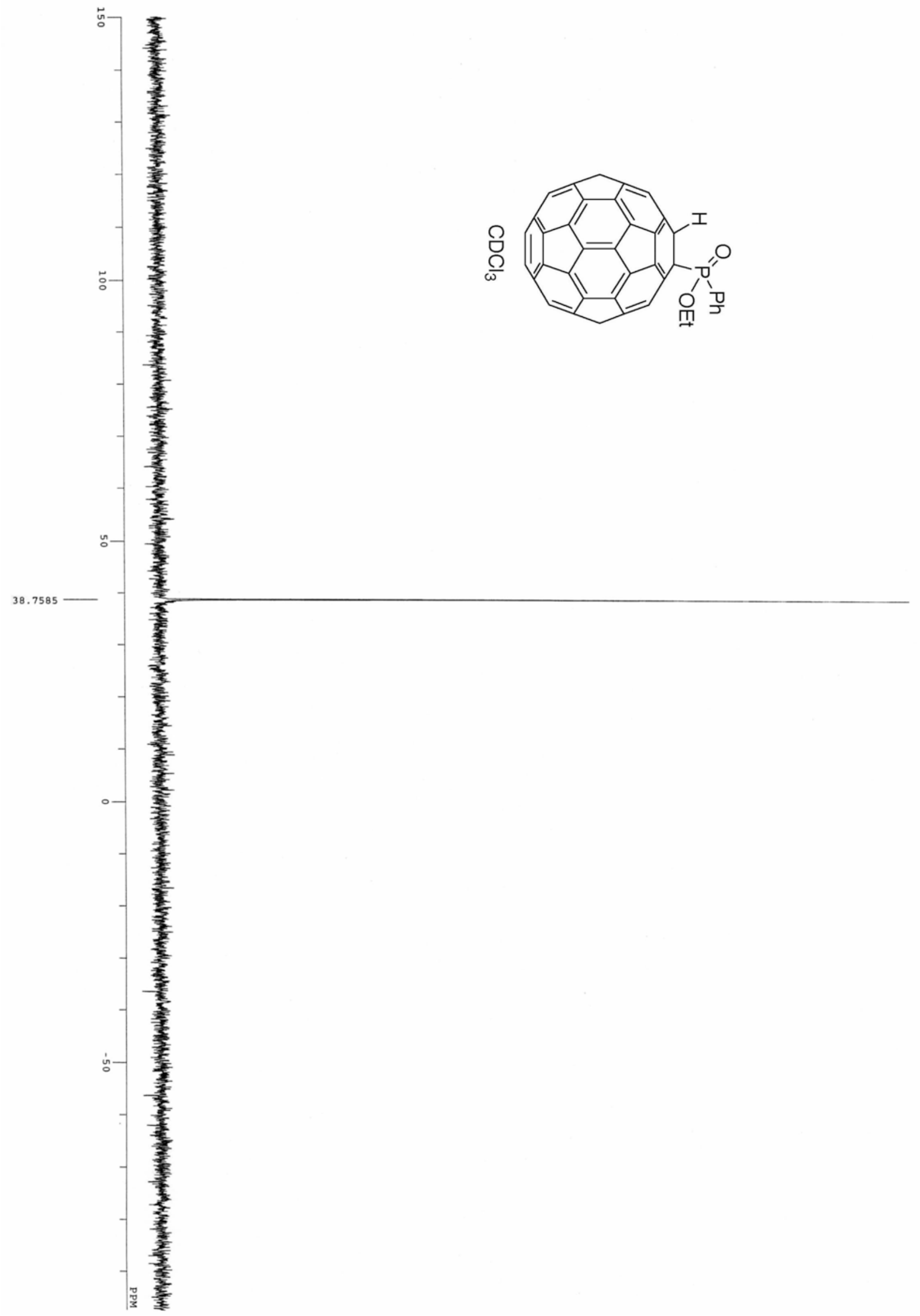


${ }^{1} \mathrm{H}$ NMR spectrum (Table 1, entry 9)

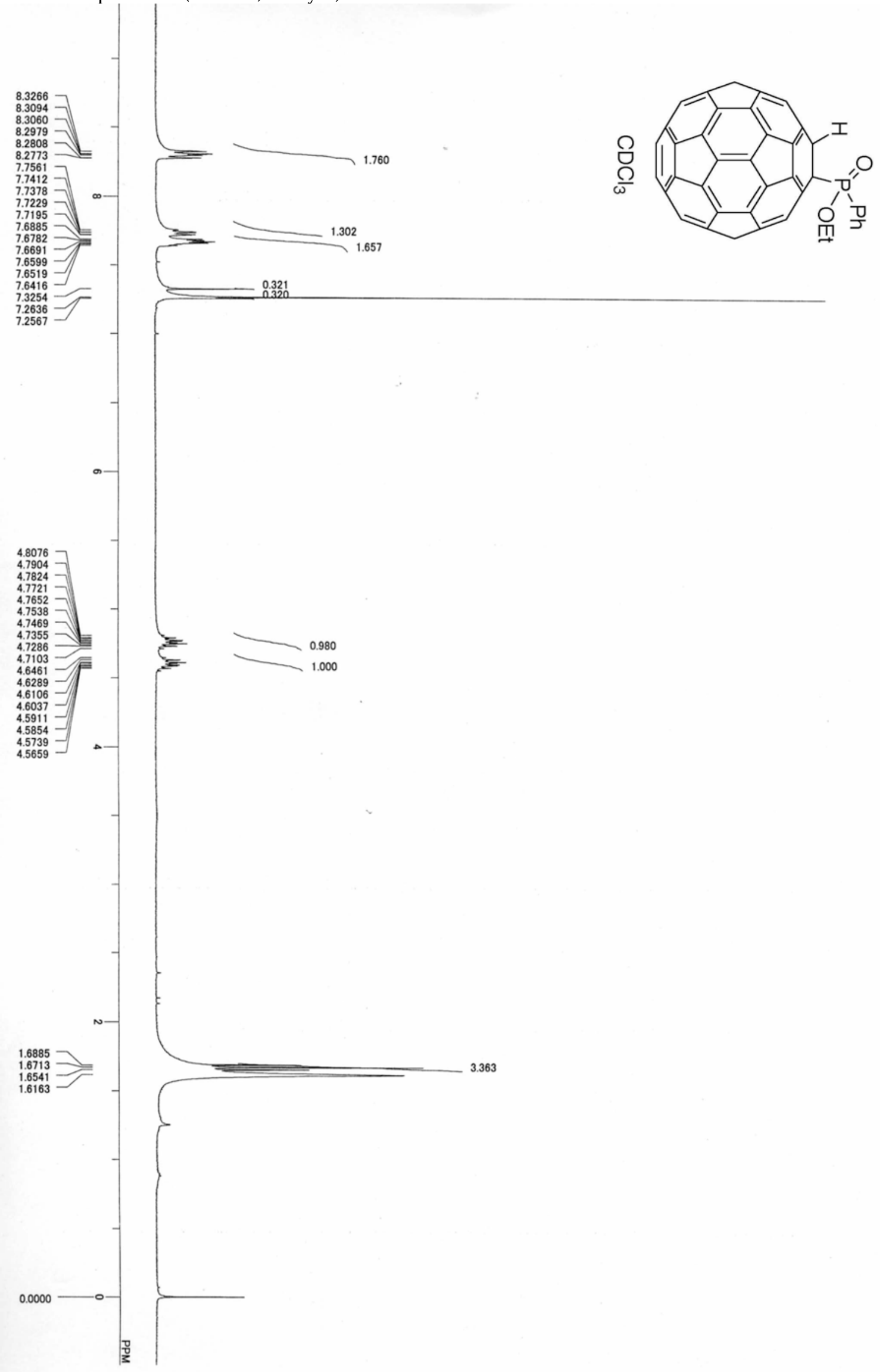


${ }^{13} \mathrm{C}$ NMR spectrum (Table 1, entry 9)

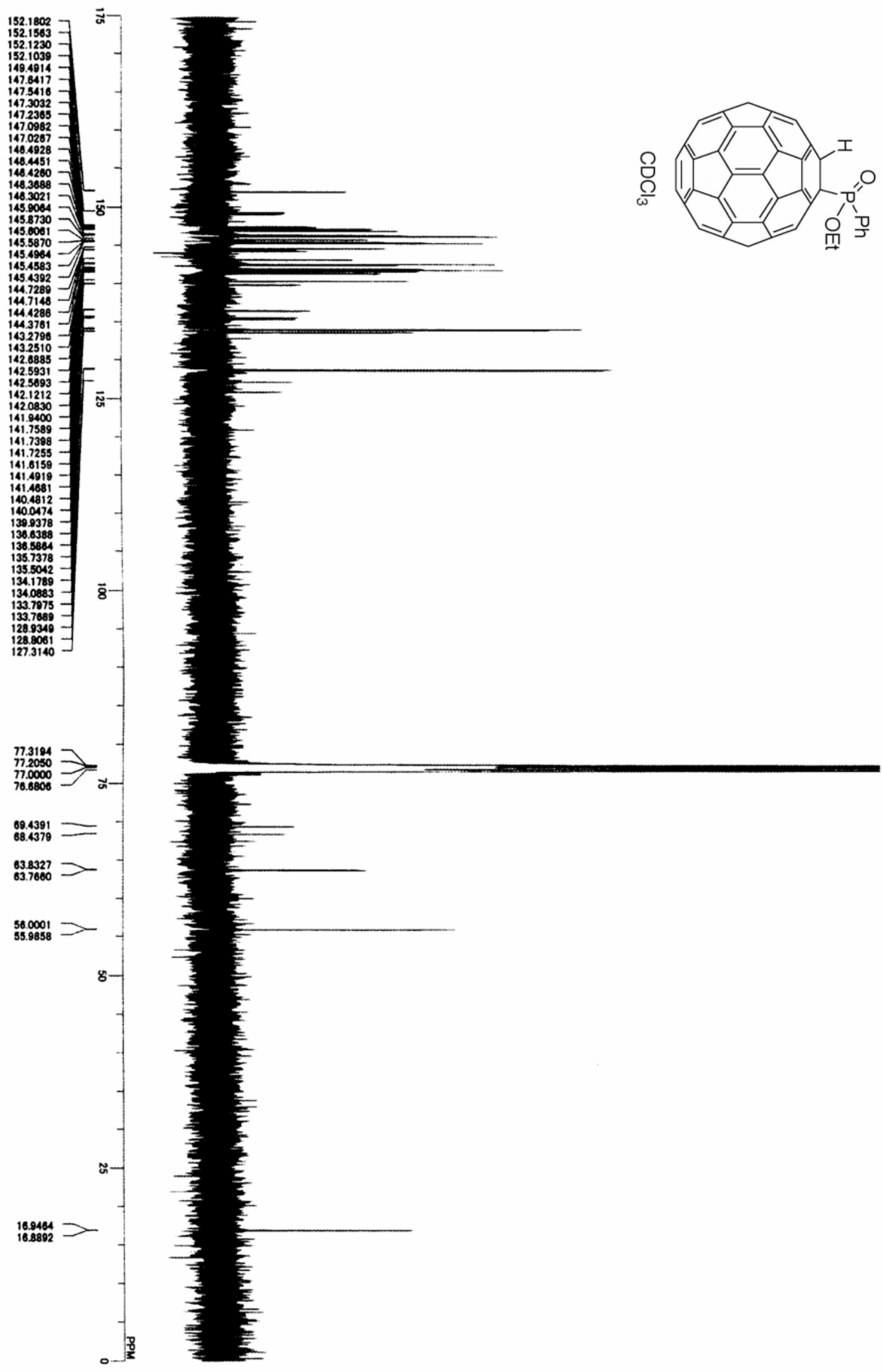


DEPT (135) spectrum (Table 1, entry 9)

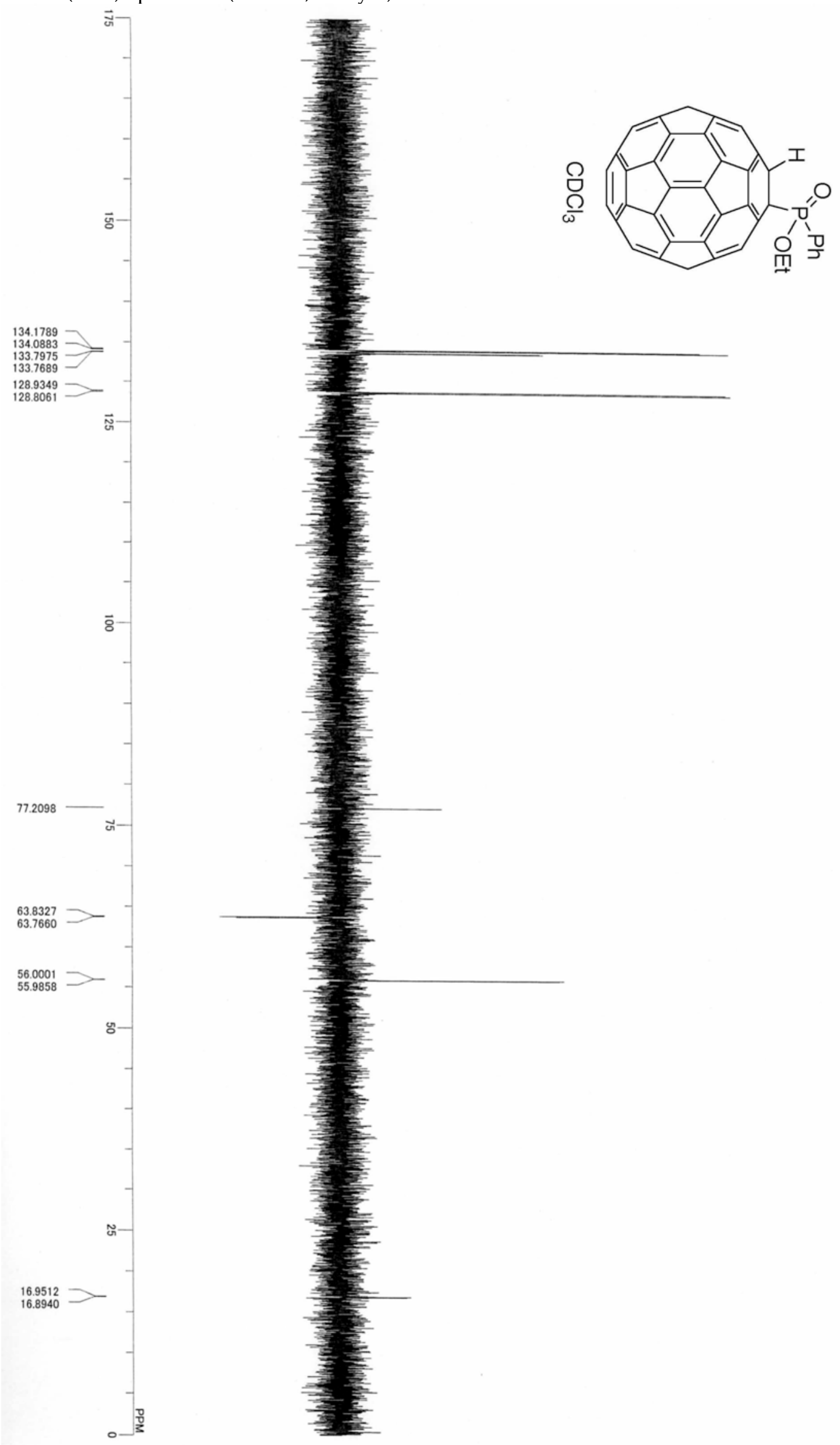


MS spectrum (Table 1, entry 9)

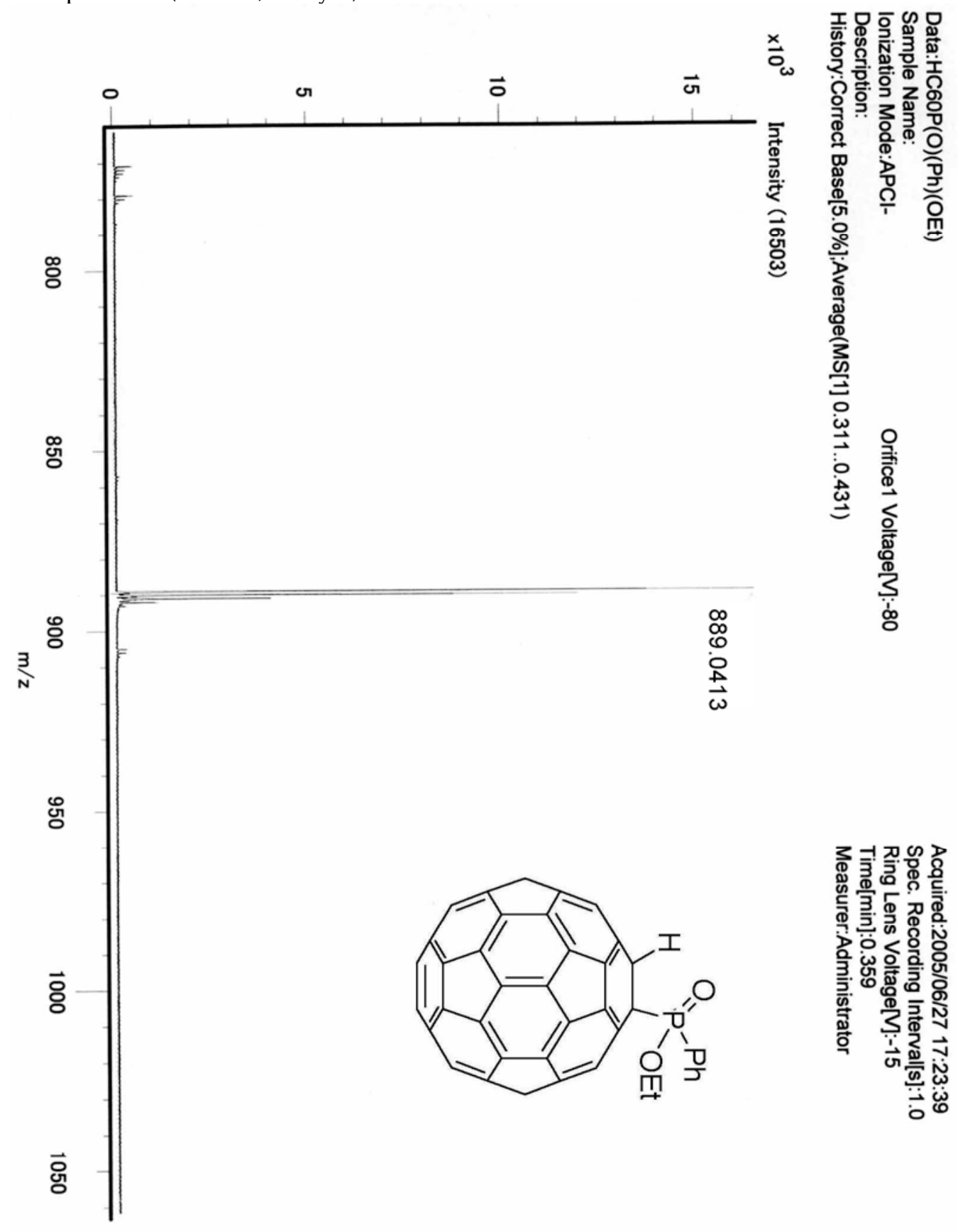


${ }^{31}$ P NMR spectrum (Table 1, entry 10)

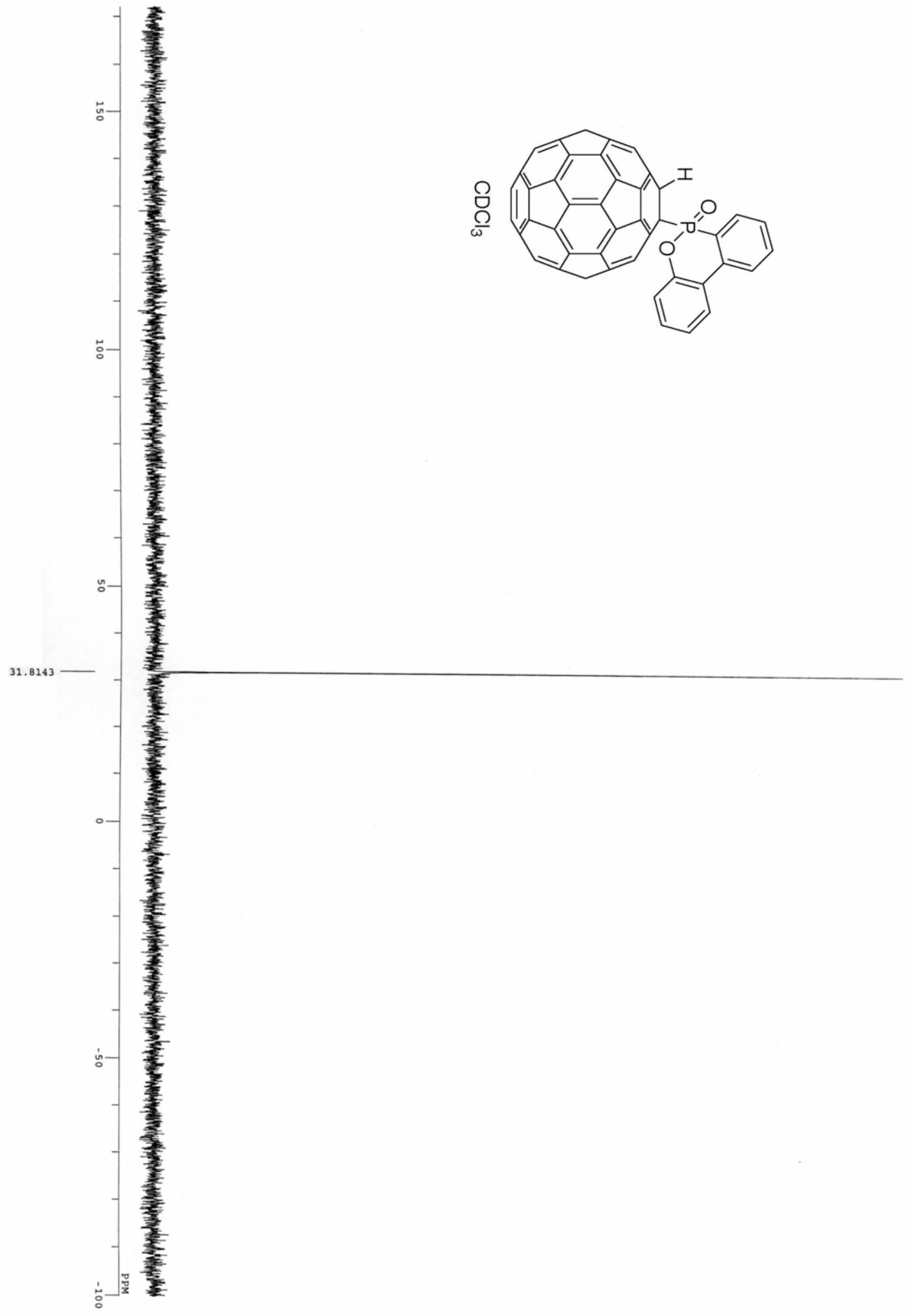


${ }^{1} \mathrm{H}$ NMR spectrum (Table 1, entry 10)

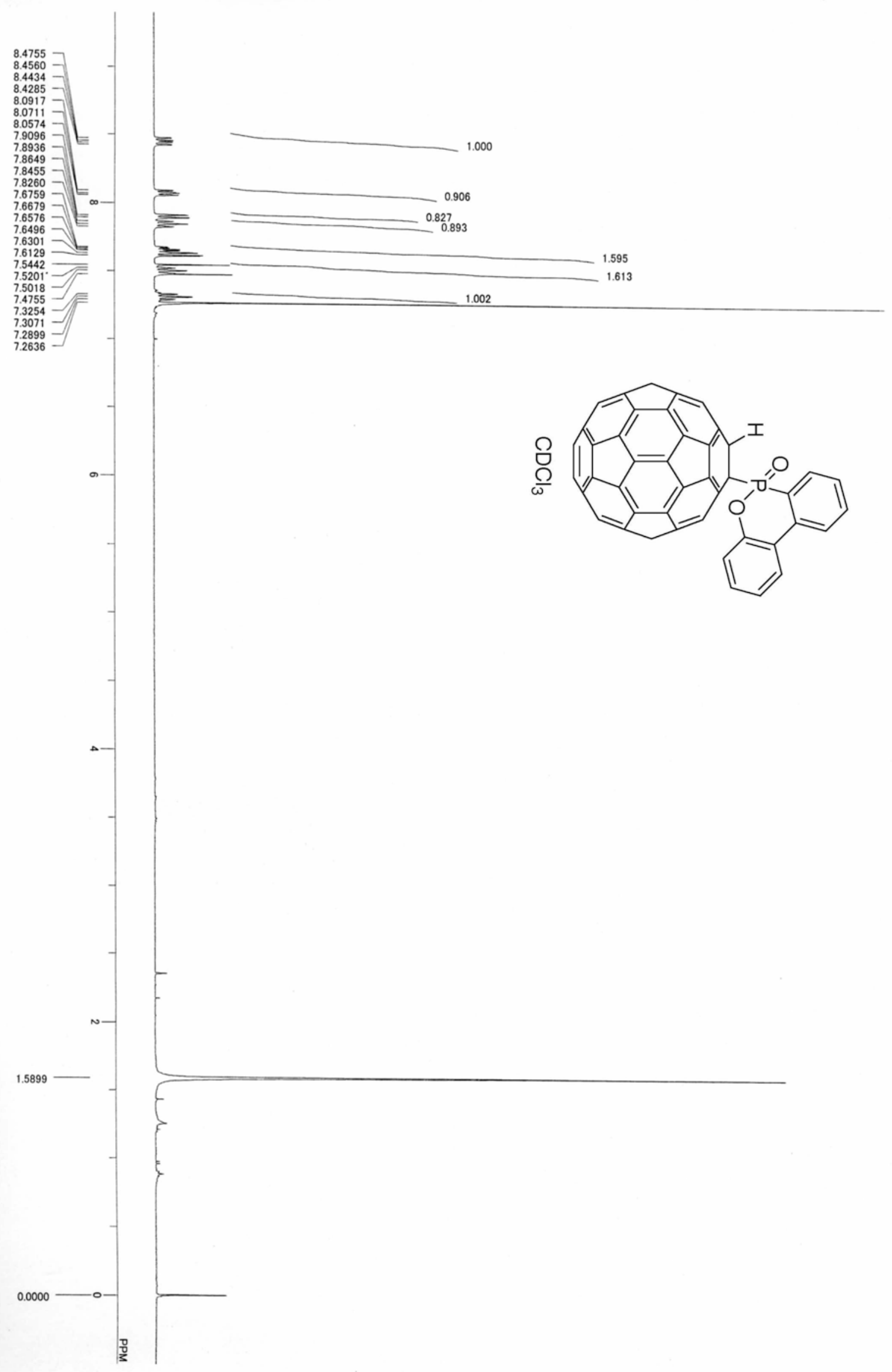


${ }^{13} \mathrm{C}$ NMR spectrum (Table 1, entry 10)

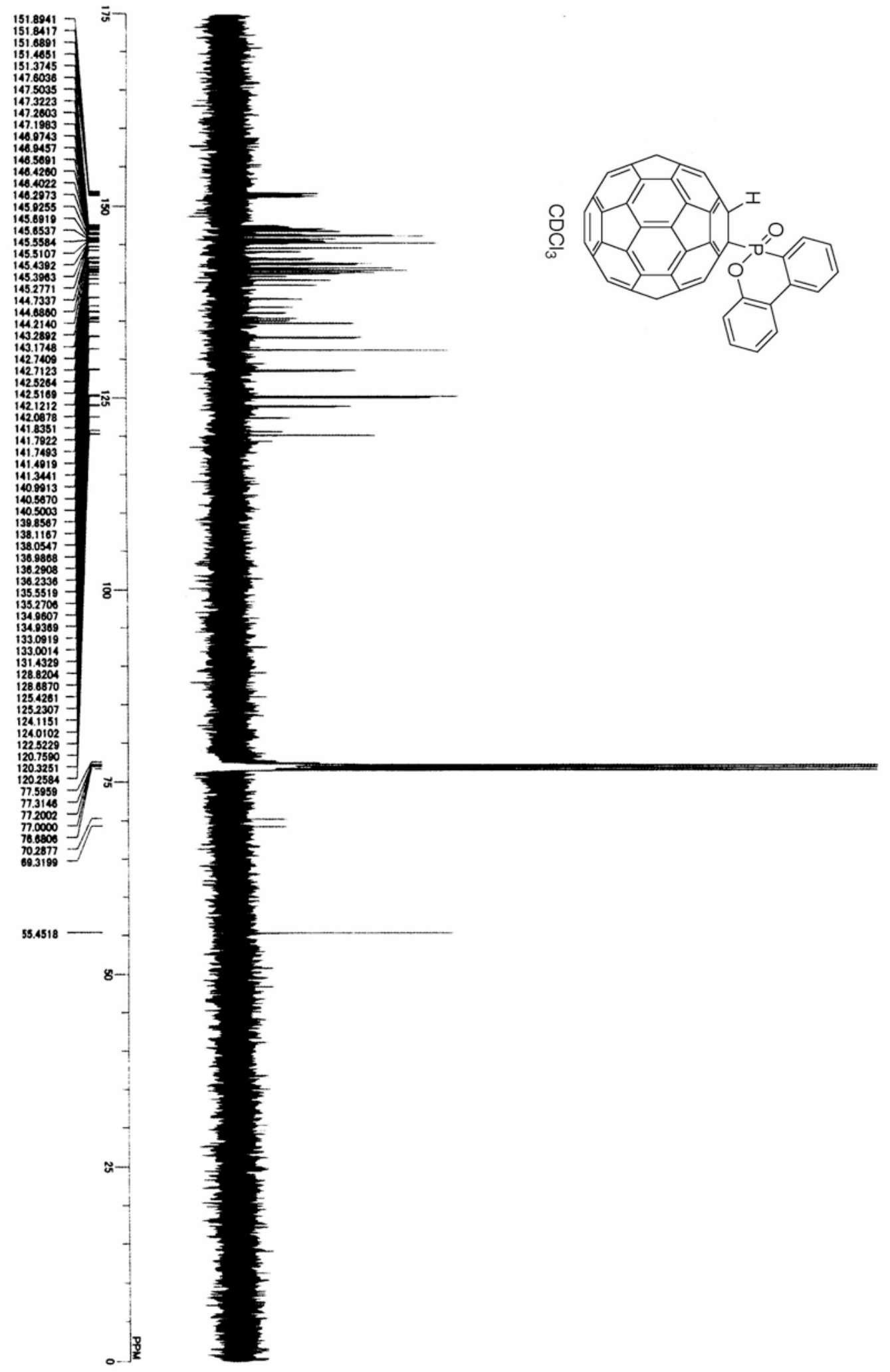

S33 
DEPT $\left(135^{\circ}\right)$ spectrum (Table 1, entry 10)

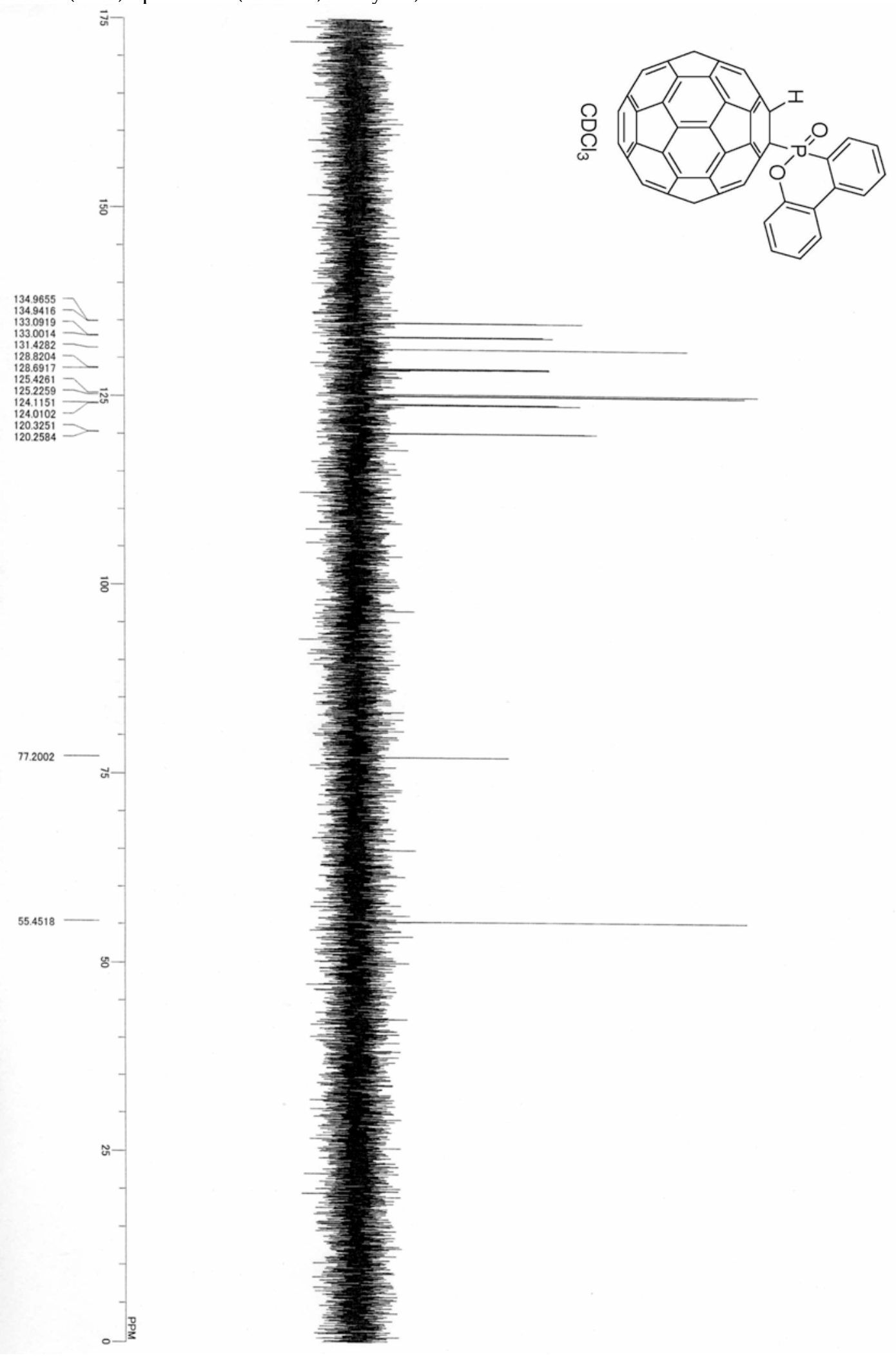


MS spectrum (Table 1, entry 10)

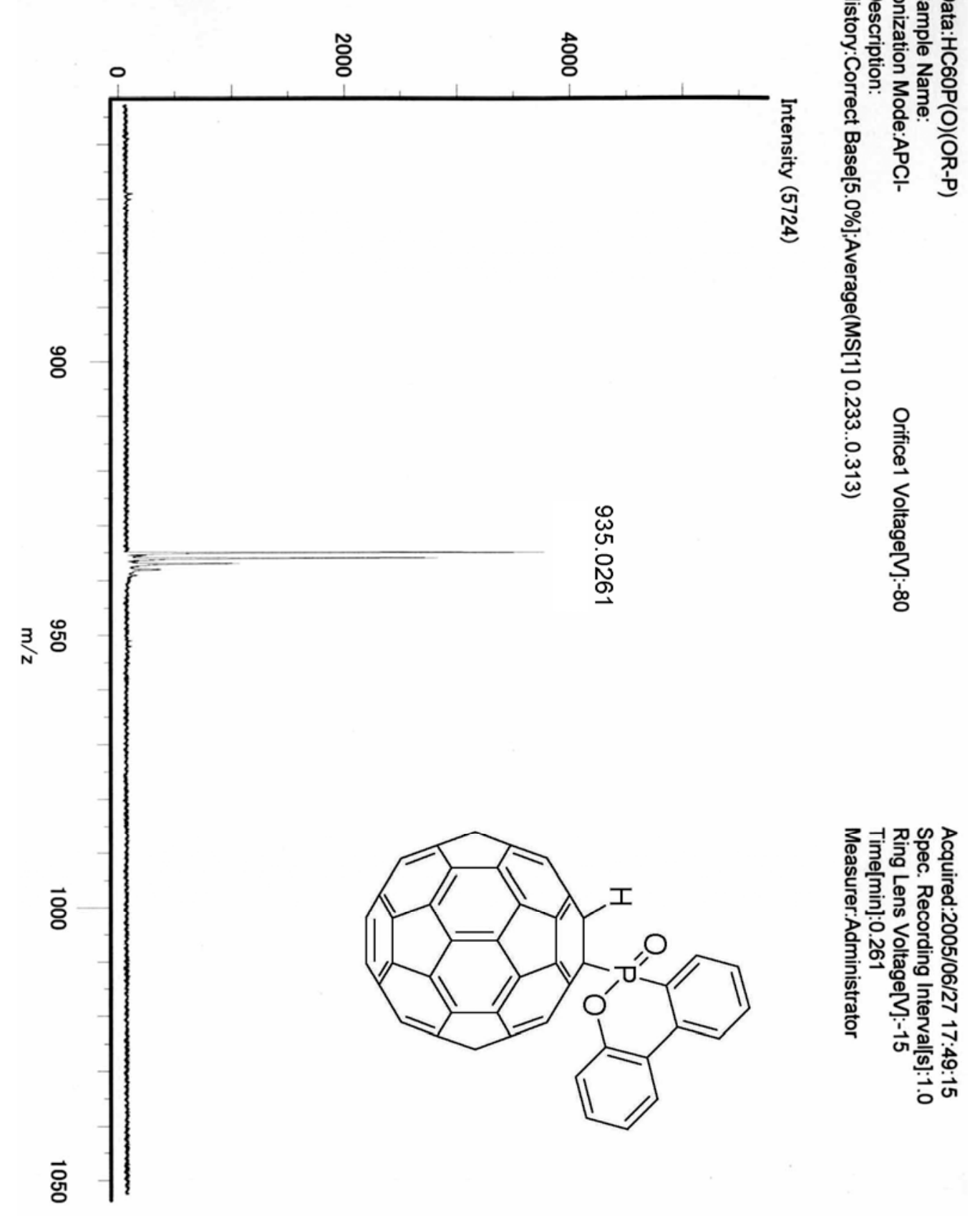


${ }^{31}$ P NMR spectrum (Table 1, entry 12)

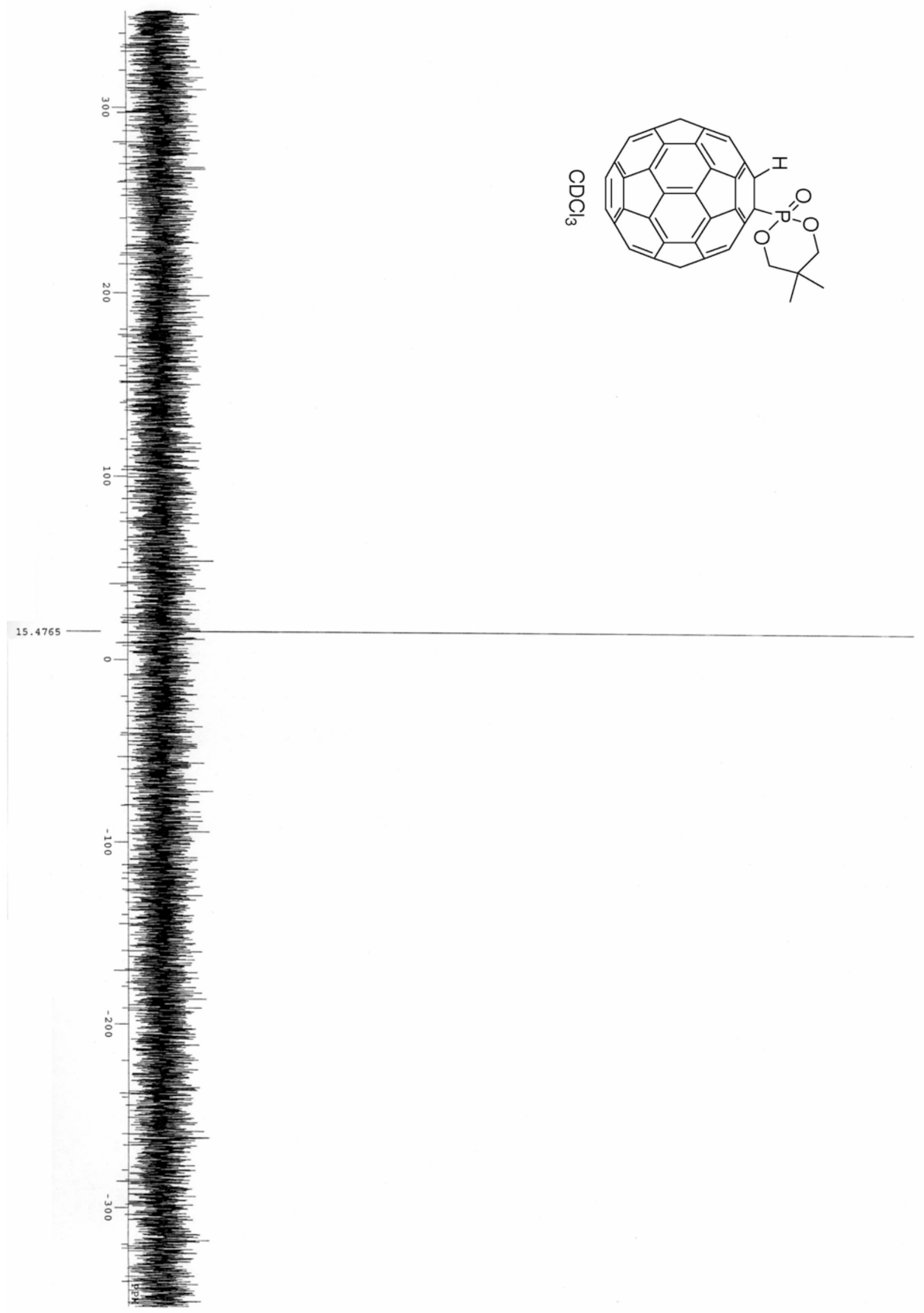


${ }^{1} \mathrm{H}$ NMR spectrum (Table 1, entry 12)

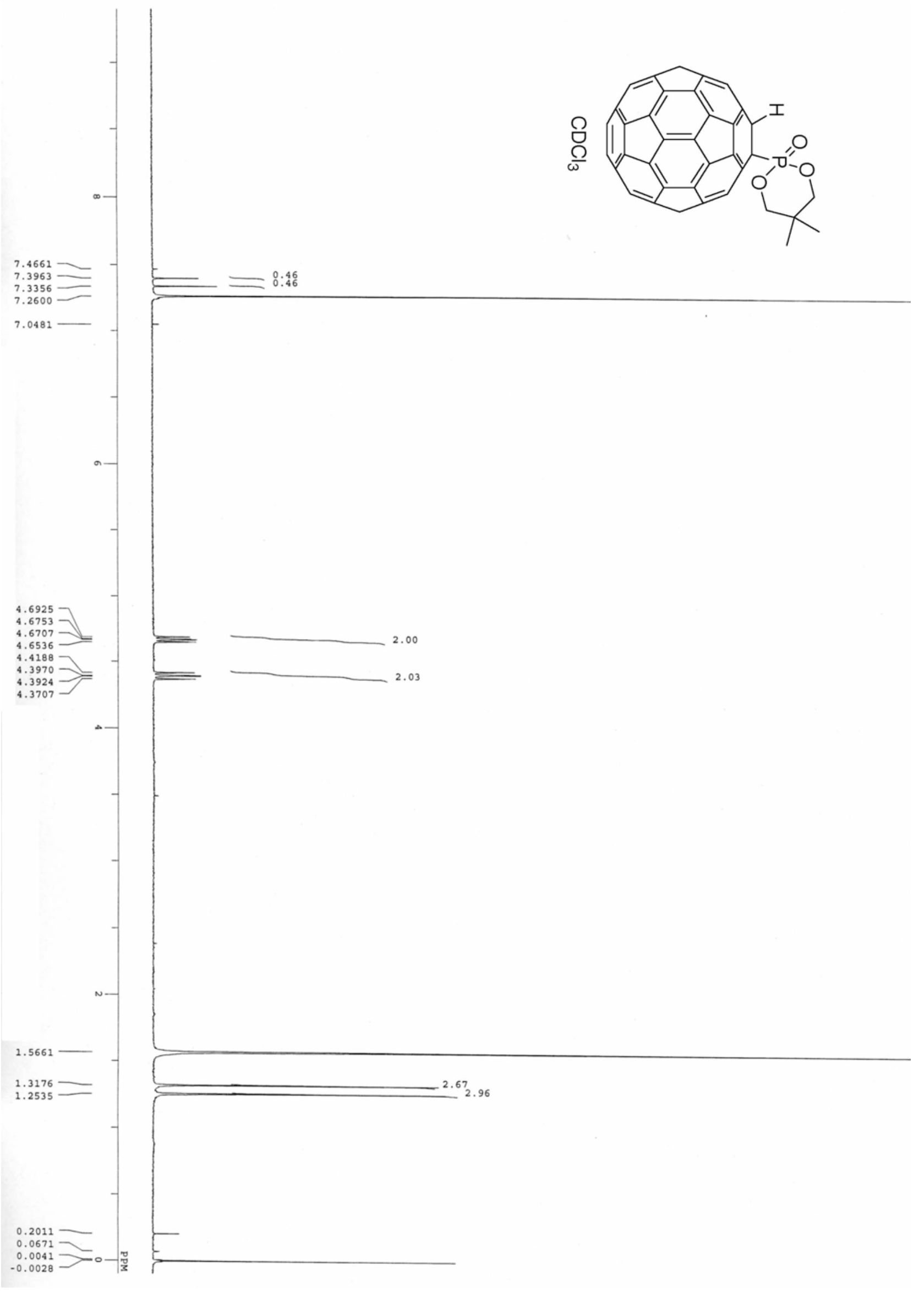


${ }^{13} \mathrm{C}$ NMR spectrum (Table 1, entry 12)

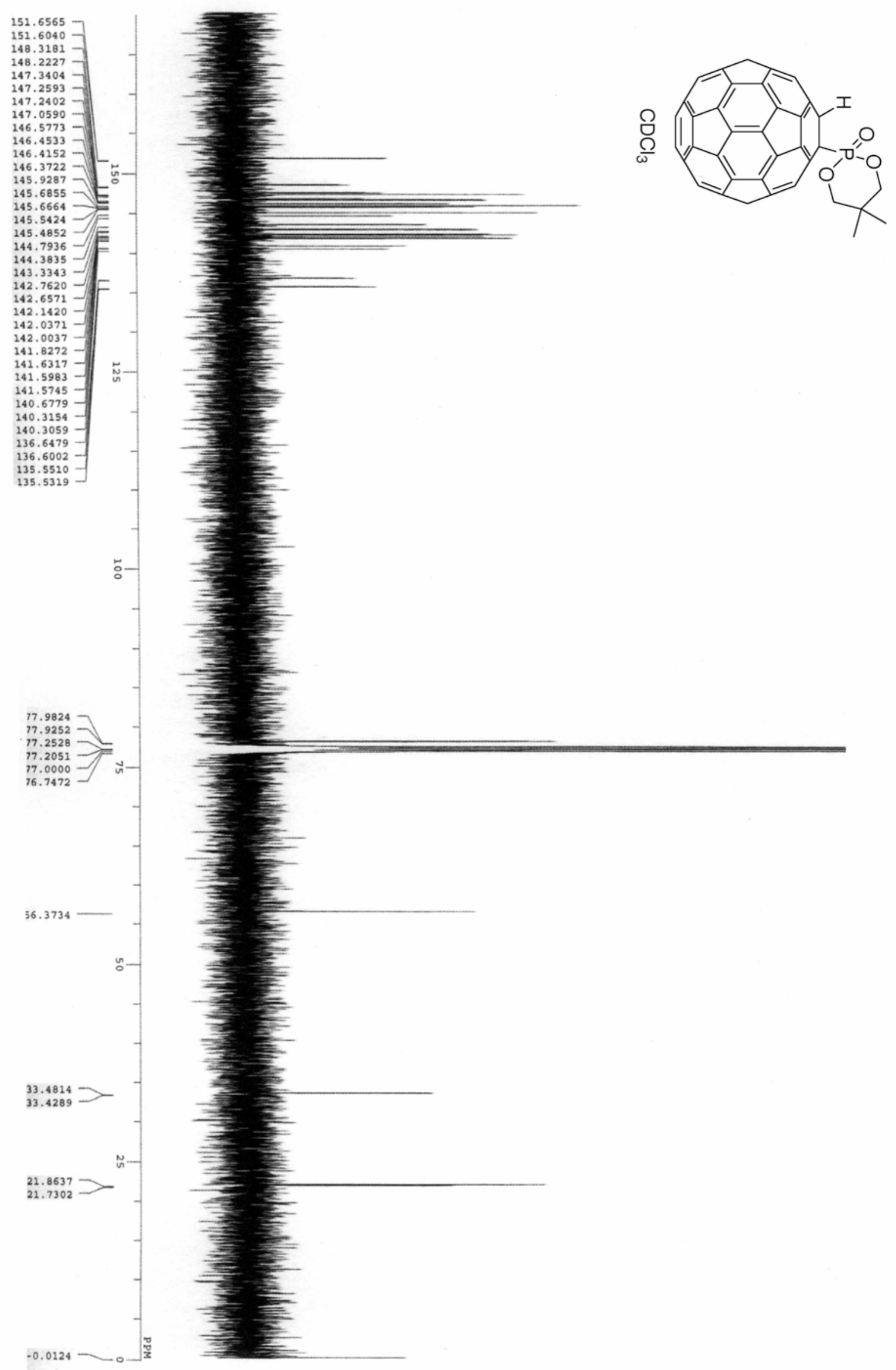


DEPT $\left(135^{\circ}\right)$ spectrum (Table 1, entry 12 )

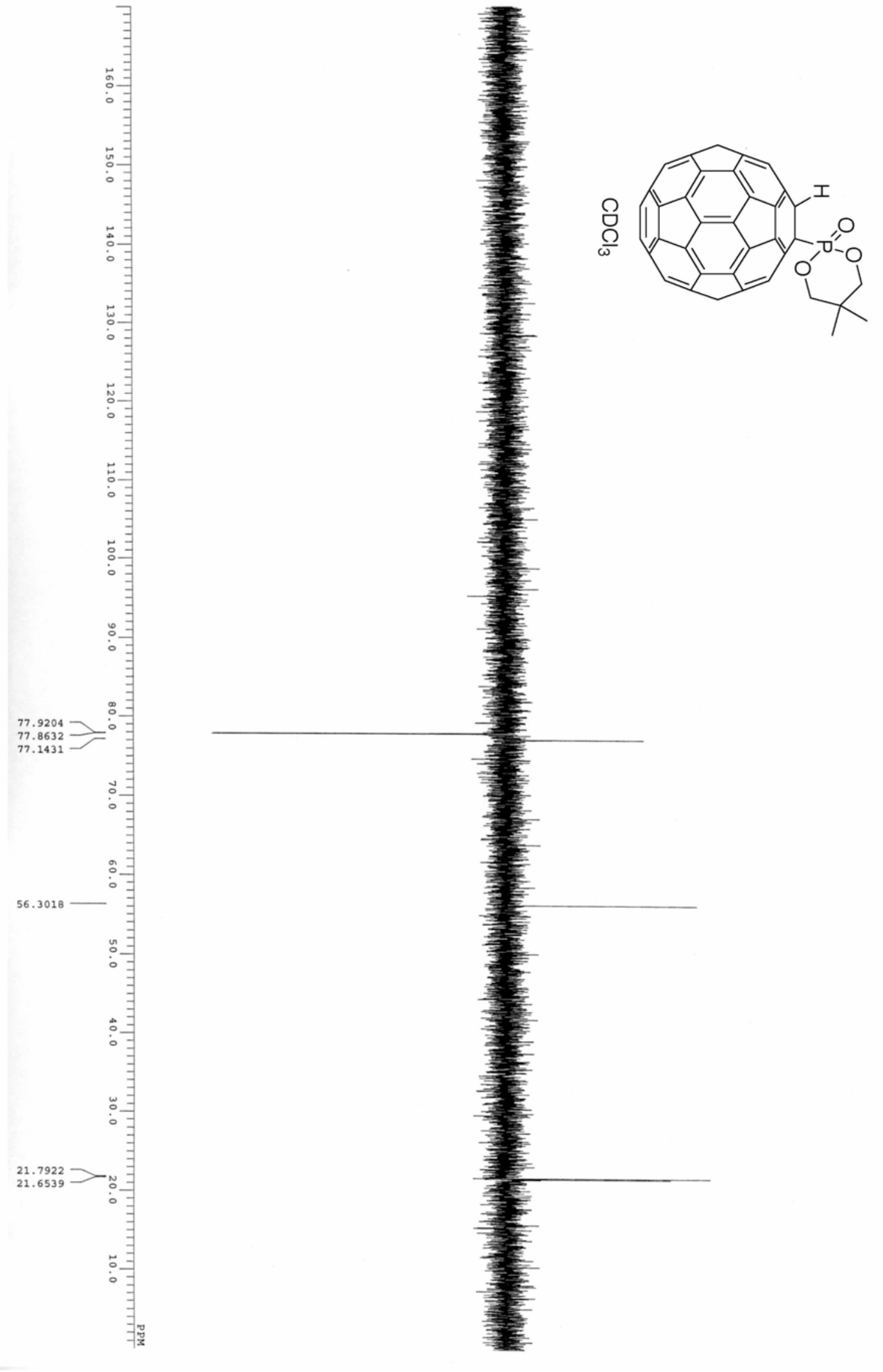


MS spectrum (Table 1, entry 12)

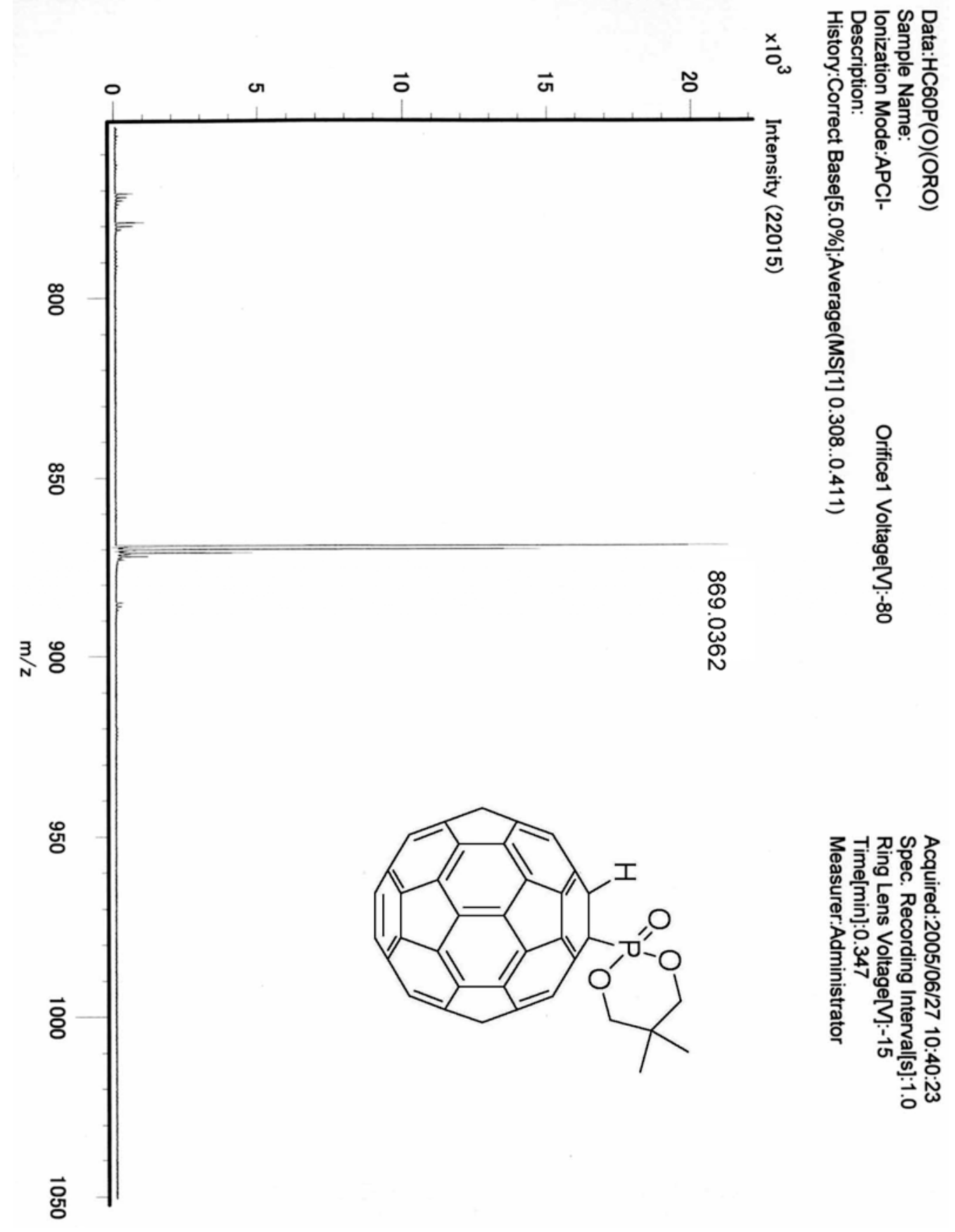

